\title{
1 Human antigen-specific memory natural killer cell responses develop against HIV-1 and influenza virus and are dependent on MHC-E restriction
}

Stephanie Jost ${ }^{1}$, Olivier Lucar ${ }^{1}$, Taylor Yoder $^{1}$, Kyle Kroll ${ }^{1}$, Sho Sugawara ${ }^{1}$, Scott Smith ${ }^{1}$, Rhianna Jones $^{1}$, George Tweet ${ }^{1}$, Alexandra Werner ${ }^{1}$, Phillip J. Tomezsko ${ }^{2}$, Haley L. Dugan ${ }^{2 \dagger}$, Joshua Ghofrani $^{1}$, Marcus Altfeld ${ }^{3}$, Adam Grundhoff ${ }^{3}$, Michaela Muller-Trutwin ${ }^{4}$, Paul Goepfert ${ }^{5}$, R. Keith Reeves ${ }^{1,2 *}$

${ }^{1}$ Center for Virology and Vaccine Research, Beth Israel Deaconess Medical Center, Harvard Medical School, Boston, MA 02115, USA; ${ }^{2}$ Ragon Institute of Massachusetts General Hospital, MIT, and Harvard, Cambridge, MA 02139, USA; ${ }^{3}$ Heinrich Pette Institute, Leibniz Institute for Experimental Virology, 20251 Hamburg, Germany; ${ }^{4}$ Institut Pasteur, HIV, Inflammation and Persistence Unit, Paris, France; 5 University of Alabama at Birmingham, Birmingham, AL 35294, USA

${ }^{*}$ Corresponding author

R. Keith Reeves

Center for Virology and Vaccine Research

21

22

Beth Israel Deaconess Medical Center

3 Blackfan Circle

23 Boston, MA 02215

24 Ph: (617-735-4586)

25 Fax: (617-735-4527)

E-mail: rreeves@bidmc.harvard.edu

${ }^{\dagger}$ Current address: Committee on Immunology, University of Chicago, Chicago, IL 60637, USA

Running Title: Human Memory NK Cells

32 Abstract: 185 words

33 Main Text: 2713 words

34 Methods Text: 2035 words

$35 \quad 5$ Figures

367 Supplemental Figures

373 Supplemental Tables

3862 references 


\section{ABSTRACT}

42 For over a decade, multiple studies have disputed the notion of natural killer (NK) cells as

43 purely innate lymphocytes by demonstrating that they are capable of putative antigen-specific

44 immunological memory against multiple infectious agents including two critical global health

45 priorities - HIV and influenza. However, the mechanisms underlying antigen specificity remain

46 unknown. Herein, we demonstrate that antigen-specific human NK cell memory develops upon

47 exposure to both HIV and influenza, unified by a conserved and epitope-specific targetable

48 mechanism firmly dependent on the activating CD94/NKG2C receptor and its ligand HLA-E, and

49 confirm these findings by three rigorous and novel assays. We validated the permanent

50 acquisition of antigen-specificity by individual memory NK cells by single-cell cloning. We

51 identified biomarkers of antigen-specific NK cell memory through RNA-Seq transcriptomic

52 fingerprints and complex immunophenotyping by 30-parameter flow cytometry showing elevated

53 expression of KLRG1, $\alpha 4 \beta 7$ and NKG2C. Finally, we show individual HLA-E-restricted peptides

54 that may constitute the dominant response in HIV-1- and influenza-infected persons in vivo. Our

55 findings clarify the mechanisms behind formation of antigen-specific memory NK cells, and

56 suggest they could be targeted for future vaccines, cure strategies, or other therapeutic 57 interventions. 


\section{INTRODUCTION}

61 Natural killer (NK) cells are lymphocytes classically considered part of the innate immune system

62 based on their ability to mediate very rapid and nonspecific responses to virally infected or

63 neoplastic cells. A plethora of studies have provided compelling evidence for the significant

64 contribution of NK cells to the immune control of HIV and influenza virus infections ${ }^{1-3}$, two of the

65 most critical global health priorities. NK cells represent a potent antiviral effector cell population

66 that can promptly respond to HIV and influenza exposure without the need for prior antigen

67 sensitization. The interest in harnessing NK cell functions for vaccine design and therapeutic

68 interventions against these pathogens has dramatically increased in recent years, largely driven

69 by a series of observations indicating subpopulations of NK cells, called memory or adaptive NK

70 cells, manifest multiple different forms of durable adaptive capabilities ${ }^{4-20}$. This includes reports of

71 true antigen-specific memory NK cells $s^{5,7,8,10,13,14,18-20}$ that can mediate recall responses against

72 multiple infectious agents including $\mathrm{HIV}^{7,13,18}$ and influenza ${ }^{7,14}$. Adaptive NK cell subsets have

73 been associated with protective effects in people living with HIV (PLWH $)^{21-24}$ and exposure to

74 influenza antigens induces protective influenza-specific memory NK cells in mouse models ${ }^{7,14}$.

75 Thus, antigen-specific memory NK cells represent a third arm of the immune system that can be

76 targeted by prophylactic or therapeutic interventions.

77 To date, only a few studies support the existence of true antigen-specific recall responses by

78 NK cells in humans, which have been described against cytomegalovirus (CMV), varicella zoster

79 virus (VZV), hepatitis A (HAV) and B (HBV) virus and bacillus Calmette-Guerin ${ }^{15,18-20,25,26}$ and the

80 opacity surrounding the mechanisms of memory NK cell formation represents a major obstacle to

81 harnessing adaptive NK cell functions. Herein, we provide proof that antigen-specific NK cell

82 memory develops upon exposure to HIV and influenza in humans, and also provide the first

83 mechanistic evidence for memory NK cell recognition of target antigens at the single-cell and

84 single-peptide level.

85 


\section{RESULTS}

87 Evidence for human NK cells mediating HIV- and influenza antigen-specific responses.

88 Clear evidence of HIV- and influenza-specific recall responses by NK cells in humans is 89 lacking. We first tested HIV antigen specificity using a modified intracellular cytokine staining

90 (ICS) assay designed to uniquely quantify CD107a+ and IFN- $\gamma+$ HIV Gag-specific peripheral

91 blood NK cells in viremic PLWH or healthy donors (Fig. 1a, Table S1). In PLWH, 0.5 to $6 \%$ of

92 NK cells were reactive against HIV Gag peptides, mirroring responses found in SIV-infected

93 rhesus macaques ${ }^{13}$, whereas in healthy donors, NK cell responses were generally undetectable

94 above background. The capacity of NK cells isolated from PLWH to specifically react against HIV

95 antigens was further confirmed using a flow cytometry-based cytotoxicity assay. To do so, we

96 assessed the killing of autologous B lymphoblastoid cell lines (BLCL) pulsed with a pool of HIV

97 Gag peptides by untouched NK cells isolated either from PLWH or from healthy donors (Fig.

98 1b, Fig. S1). Significant anti-HIV Gag activity by NK cells could only be detected in PLWH,

99 while killing of BLCL pulsed with a pool of EBV, CMV and influenza peptides was comparable

100 between PLWH and healthy individuals. Potent lysis of MHC-devoid K562 cells, a target cell

101 line devoid of MHC commonly used to evaluate NK cell cytotoxicity and that triggers non

102 antigen-specific stimulation, was similarly achieved by NK cells from PLWH and HIV-negative

103 donors. PLWH included patients on combination antiretroviral therapy (cART) with

104 undetectable viral loads, untreated viremic PLWH, as well as HIV elite controllers who achieve

105 spontaneous control of viral replication in the absence of treatment (HIV RNA levels $<50$

106 copies $/ \mathrm{mL}$ for at least a year) (Table S1) ${ }^{27}$. NK cells from elite controllers with detectable HIV

107 gag-specific cytotoxic activity displayed the most potent responses observed. These data

108 showed that NK cells that can specifically respond to HIV antigens develop in PLWH and

109 mediate potent anti-HIV activity, suggesting HIV-specific NK cells might significantly contribute

110 to control of HIV. Using a similar ICS-based assay as described above, we next measured the 
111 ability of human NK cells to mediate antigen-specific responses to influenza and CMV, to which

112 most humans have pre-existing immunity memory. Enriched NK cells from donors were

113 stimulated with peptide pools derived from the conserved matrix protein 1 (MP1) and

114 nucleoprotein (NP) of the $2009 \mathrm{H} 1 \mathrm{~N} 1$ pandemic strain (A/California/08/2009 and

115 A/California/04/2009, respectively) or derived from CMV pp65 (Fig. 1c). We detected influenza

116 positive NK cell responses in $45 \%$ of HIV-negative donors who provided blood samples between

1172017 and 2020. Altogether, these results suggested that peripheral blood NK cells can mediate

118 antigen-specific recall responses in humans, a hallmark of memory.

\section{Single-cell NK cloning confirms antigen-specificity of memory NK cells}

121 To further investigate the existence of antigen-specific memory NK cells that can efficiently

122 respond to HIV and influenza and study these likely rare cells in more detail, we clonally

123 expanded individual peripheral blood NK cells from 20 PLWH (4 cART-treated and 16 viremic

124 untreated) and 8 healthy adults (Fig. S2). Using a cytotoxicity assay, we assessed the ability of

125 single NK cell clones (NKCL) to lyse MHC-devoid K562 cells (to confirm normal NK cell function)

126 or autologous BLCL pulsed with a pool of self-peptides, CMV pp65-, HIV Gag-, HIV Envelope

127 (Env)-, A/California/04/2009 H1N1 NP- and A/California/08/2009 H1N1 MP1-derived

128 overlapping peptides (Fig. 2). As expected, NKCL could potently lyse K562 cells and had little to

129 no reactivity to BLCL pulsed with a self-peptide control. Approximately $29 \%$ of NKCL from PLWH

130 (Fig. 2a) and 45\% of NKCL from healthy adults (Fig. 2b) displayed positive responses against

131 HIV or influenza, respectively. While the magnitude of HIV-specific responses did not differ

132 between treated and untreated PLWH (Median \% Gag-specific killing, cART-treated: 6, viremic:

133 4.8, $p=0.43$; median \% Env-specific killing, cART-treated: 10, viremic: 7.4; $p=0.35)$, HIV-specific

134 NKCL were more frequently detected in untreated viremic participants (\% HIV-specific NKCL,

135 CART-treated: 20, viremic: 32 ). Strikingly, we were able to isolate NKCL with very robust anti-HIV

136 (up to $87 \%$ specific lysis against Env) and anti-influenza H1N1- (up to $57 \%$ specific lysis against 
137 NP) cytotoxic activity. Interestingly, 30\% of NKCL displaying over $6 \%$ specific killing against HIV

138 Gag or Env were able to respond to both antigens, with NKCL presenting the highest responses

139 (over 30\% specific killing) being able to respond to either Gag or Env but never both. Together

140 these data provide the first proof of HIV-and influenza-specific NK cells in humans and show that

141 NK cells displaying true and robust antigen-specific killing are found at the single cell level.

143 Mechanisms of memory NK cell target recognition and killing are dependent on the

144 activating NKG2C receptor

145 To define transcriptional profiles associated with antigen-specific NK cell responses, we first

146 performed RNAseq analysis on $8 \mathrm{NKCL}$ with anti-HIV activity and $7 \mathrm{NKCL}$ that did not react

147 against HIV from the same individuals. Overall, 12 genes were significantly upregulated and 4

148 genes downregulated in HIV Env/Gag-specific NKCL (Fig. 3a, Fig. S3). Of interest, HIV-specific

149 NKCL expressed NLRP7 and GSTM2, which are involved in cellular defense responses, as well

150 as genes involved in regulation of the actin cytoskeleton, such as FRMD5, CDC42EP4,

151 PLEKHG2, and in metabolic functions, such as RGPD8 and Ak5. Pathway enrichment analysis

152 supported clear changes in metabolic pathways in HIV-specific NKCL. Overall, our analysis

153 revealed transcriptional profiles consistent with maturation and activation that are associated with

154 memory NK cells endowed with potent anti-HIV activity, but also identified a transcriptional profile

155 that may be unique to this cell type.

156 To further define the subset of peripheral blood NK cells that drives antigen-specific memory

157 responses, we then compared expression levels of 21 cell surface receptors between NKCL with

158 anti-HIV Env/Gag activity and those that did not react to those HIV antigens using advanced

159 multiparameter flow cytometry (Table S2). Phenotypic analysis revealed that most HIV-specific

160 NKCL express activating (i.e, NKp46, NKG2D, NKG2C) and inhibitory (NKG2A, CD85j) receptors

161 typically found on NK cells, trafficking markers (i.e., $\alpha 4 \beta 7$, CXCR6, CCR5, CCR7), co-stimulatory

162 molecules (i.e., 2B4, CD2, CD8) and various levels of maturation/activation markers (CD57, 
163 KLRG1, PD1, Tim-3, HLA-DR) or KIRs (Fig. S4). Compared to NKCL that did not react to HIV,

164 HIV-specific NKCL had enhanced expression of KLRG1 and $\alpha 4 \beta 7$ and lower expression of CCR5

165 (Fig. 3b). Furthermore, analysis using non-linear dimensionality reduction algorithm (t-SNE)

166 revealed distinct clustering for HIV reactive and non- HIV-reactive NKCL, with HIV-reactive NKCL

167 being partly associated with cell clusters expressing high levels of the inhibitory NKG2A or high

168 levels of activating NKG2C and/or inhibitory KIR3DL1 (Fig. 3c and Fig. S5).

NKG2C is an activating NK cell receptor that forms heterodimers with CD94 and interacts with

170 the nonclassical MHC class I molecule HLA-E bound to HLA-E-stabilizing peptides. High cell

171 surface expression of NKG2C/CD94 has been consistently linked to long-lived NK cells endowed

172 with adaptive capabilities that develop upon human CMV infection ${ }^{11,28-31}$. Variants of CMV UL-40-

173 derived peptides that mimic canonical HLA class-I-derived leader peptides are presented by HLA-

174 E and finely tune adaptive NKG2C+ NK cell functions ${ }^{15,26}$, suggesting HCMV-specific recognition

175 via the NKG2C/HLA-E axis. Moreover, we previously showed that in non-human primates, HIV

176 antigen-specific memory NK cell responses largely depend on NKG2A/C ${ }^{13}$. As HIV-specific NKCL

177 were associated with high NKG2C expression (Fig. 3c), we directly assessed the role of NKG2C

178 in HIV antigen-specific memory NK cell responses. HIV Env-specific NKCL were co-cultured with

179 BLCL pulsed with HIV Env peptides in the presence of blocking antibodies against activating

180 receptors and specific lysis measured (Fig. 3d, Fig. S6a). NKG2C blockade significantly

181 decreased HIV Env-specific responses by NKCL, while blockade of natural cytotoxicity receptors

182 did not or only marginally impacted HIV-specific NK cell responses, corroborating our previous

183 findings ${ }^{13}$. Accordingly, we found a positive correlation between influenza-specific killing by

184 NKCLs and the proportions of NKG2C+ NKCL $(p=0.0007, r=0.5090)$ and NKCL clones

185 displaying over $10 \%$ specific killing against influenza antigen had significantly higher NKG2C

186 expression than NKCLs that did not or only marginally reacted against influenza (Fig. 3e, Fig.

187 S6b). Altogether, our results confirm that antigen-specific memory NK cell responses are 188 dependent on NKG2C, likely indicative of an HLA-E-dependent recognition mechanism. 
190 HIV- and influenza-derived peptides bind HLA-E, the ligand for NKG2C, and potently

\section{1 activate antigen-specific NK cells.}

192 HLA-E displays limited polymorphism and presents largely conserved nonameric peptides

193 derived from leader sequences of other HLA class I molecules. However, pathogen-derived

194 peptides ${ }^{32-35}$, including HIV Pol, Vif and p24 ${ }^{36-38}$ have been shown to bind HLA-E. To establish

195 the relevance of an HLA-E-dependent recognition mechanism in antigen-specific memory NK

196 cell responses against two unrelated viruses, we first screened for canonical peptides derived

197 from HIV Env, HIV Gag and influenza NP that can stabilize HLA-E at the surface of K562 cells

198 stably expressing HLA-E*0101 (Fig. 4a, Fig. S7, Table S3). Among all tested nonameric

199 peptides (84 for HIV Env, 60 for HIV Gag, 23 for influenza NP), two HIV Env-derived and two

200 influenza NP-derived peptides were identified that could stabilize HLA-E either to the same

201 extent as the positive control (CMV UL40 VMAPRTLIL) or up to two-fold greater.

202 To evaluate the effect of HLA-E-binding peptides on antigen-specific NK cell function, we

203 compared killing by NKCL from 5 PLWH of BLCL pulsed with pools of 15-mer peptides with 11

204 amino acids overlap covering the whole HIV Gag and HIV Env with that of BLCL pulsed with

205 single HLA-E-binding peptides, including HIV Env-derived VLKYWWNLL and ILPCRIKQI or HIV

206 Gag AISPRTLNA, a peptide that has been previously shown to significantly bind HLA-E and affect

207 NK cell function ${ }^{36,37}$ (Fig 4b). 4 out of 11 NKCL (36\%) and 5 out of 6 NKCL (83\%) reacted to

208 single HLA-E-binding HIV Gag- and HIV Env-derived peptides (range of positive responses twice

209 above the background: 6-21\% Gag-specific and 6-22\% Env-specific killing). Similarly, we

210 evaluated killing of BLCL pulsed with either pools of 15-mer overlapping peptides encompassing

211 the whole NP sequence or single TMDSNTLEL and FLARSALIL peptides (all derived from

212 A/California/7/2009 H1N1), by 20 NKCL from 5 healthy donors (Fig. 4c). Thirteen NKCL (65\%)

213 reacted to TMDSNTLEL, among which 6 also displayed responses against the NP pool, two of

214 these being also able to respond to FLARSALIL (range of positive responses twice above the 
215 background: 3-29\% NP-specific killing). These results show that HIV Env- and influenza NP-

216 derived nonamers can bind HLA-E and potently activate patient-derived memory NKCL.

217 Next, we sought to confirm the in vivo relevance of the novel peptide responses we 218 identified among single-cell clones. First, we showed stimulation with newly identified HIV Env219 and influenza NP-derived HLA-E-binding peptides alone promotes CD107a upregulation on a 220 subset of primary NK cells in PLWH and healthy donors, respectively (Fig. 5). CD107a up-

221 regulation in NK cells correlated significantly with NK cell-mediated cytotoxicity ${ }^{39,40}$ and in

222 NHPs, we also found a correlation between HIV-specific killing by memory NK cells and CD107a

223 up-regulation by memory NK cells. Thus, our results strongly indicate in vivo activation of

224 cytotoxic antigen-specific adaptive NK cells by single HLA-E binding nonamers. Collectively,

225 our work provides the first recognition mechanism underlying antigen-specific recall responses

226 mediated by NK cells in humans, which have the potential to be harnessed for vaccine design

227 or other therapeutic interventions.

\section{DISCUSSION}

230 Thus far, antigen-specific memory responses mediated by NK cells in humans have been

231 described in VZV-experienced adults ${ }^{18}$ as well as in HBV-infected or HAV/HBV-vaccinated

232 individuals ${ }^{19,20}$. While the idea of primate NK cell memory has become well-accepted among

233 immunologists, until now there have been no reported mechanisms describing how NK cells

234 recognize and distinguish individual antigens. Our study provides the first mechanistic evidence

235 of human NK cell memory against pathogens of global public health importance that cannot

236 currently be efficiently prevented by vaccination. We show that HLA-E possesses the previously

237 unappreciated ability to bind HIV and influenza peptides and activate virus-specific NK cells. HLA-

238 E has gained significant attention in the vaccine field as broad Mamu-E restricted $\mathrm{CD}^{+} \mathrm{T}_{\text {cell }}$

239 responses have been implicated as immune correlates of protection in macaques vaccinated with

240 a cytomegalovirus-vectored SIV vaccined ${ }^{41,42}$, and this overall function may be conserved 
241 between rhesus macaque Mamu-E and human HLA-E ${ }^{43}$. Vaccine strategies that mobilize HLA-E

242 could induce HIV- or influenza-specific memory NK cells that recognize broad epitopes across

243 viral antigens. One major issue for the development of an HIV vaccine or a universal influenza

244 vaccine is the considerable variability of viral strains circulating among humans. Antigen-specific

245 NK cell responses relying on activation of the NKG2C/HLA-E axis is in accordance with the fact

246 that in our studies, some antigen-specific NKCL were able to potently react to several antigens

247 from the same as well as from different viruses. Further, we present primary HLA-E-restricted

248 responses for both pathogens which may represent dominant memory NK cell responses in vivo

249 that could be further targeted. This feature could be highly beneficial for vaccine design and allow

250 a limited number of memory NK cells to target a broader range of HIV and influenza strains than

251 memory T cells.

252 In addition to the lack of mechanism previously described for NK cell memory, the field also

253 continues to search for comprehensive biomarkers to distinguish adaptive subpopulations of NK

254 cells from classical NK cells. CD49a, CXCR6, NKG2C, and suppressed gamma signaling chain

255 have also been implicated to partially delineate these populations, but particularly for true antigen-

256 specific memory NK cells these phenotypes maybe be incomplete ${ }^{44}$. Herein, we describe multiple

257 phenotypes that advance biomarker usage for NK cell memory. KLRG1 has been described as

258 a marker of NK cell activation ${ }^{45}$, which defines subsets of memory-like NK cells with protective

259 functions against Mycobacterium tuberculosis ${ }^{46}$ and subsets of NK cells that mediate antigen-

260 specific memory responses against HBV antigens ${ }^{19}$. Upregulation of KLRG1 on HIV-specific

261 NKCL further suggest KLRG1 might be a useful biomarker to identify antigen-specific memory NK

262 cells among bulk NK cells. Additionally, $\alpha 4 \beta 7$ was found to be upregulated on HIV-specific NKCL

263 and this corroborates previous observations that HIV and SIV infection specifically mobilizes

264 mucosal-homing NK cells ${ }^{47,48}$. Not surprisingly, due to our mechanism described in these studies

265 and others, NKG2C was also highly expressed on memory NK cells. However, antigen-specific

266 memory NK cell responses likely do not solely depend on the NKG2C/HLA-E axis, and other 
267 pathways may be additive or alternative to this mechanism. In particular, activating KIRs

268 represent another important family of receptors that may regulate memory NK cell function.

269 Specific KIR genes expressed in conjunction with their HLA ligands have been clearly associated

270 with improved HIV, HCV, CMV, flavivirus, and bacterial infection control ${ }^{49-52}$, and while the

271 interaction of inhibitory KIRs with HLA class I ligands has been studied in detail, ligands for most

272 activating KIRs remain elusive. Besides triggering NK cell activation through interactions between

273 HLA class I ligands and activating NK cell receptors, influenza- or HIV-derived peptides may

274 also disrupt inhibitory signals mediated through NKG2A or other inhibitory molecules and HLA-

275 E or their cognate HLA class I ligand, respectively ${ }^{37,53-57}$, further promoting NK cell responses.

276 This possible mechanism is consistent with HIV-reactive NKCL being partly associated with

277 high expression levels of the inhibitory NKG2A and KIR3DL1 receptors (Fig. 3c and Fig. S5).

278 Future studies beyond the scope of this work will be required to determine if KIR mediated

279 recognition is complementary or independent to this highly dominant HLA-E dependent

280 mechanism.

281 Finally, various mechanisms have been proposed to explain the spontaneous ability to 282 maintain undetectable viral loads in the absence of cART in HIV elite controllers. Notably, specific 283 variations in the human HLA class I gene locus, highly functional CD8 T cell and NK cell 284 responses and distinct configuration of the proviral reservoir have been associated with enhanced 285 virological control ${ }^{58-61}$. Our data now suggest enhanced HIV-specific NK cell cytotoxicity as a 286 potential correlate of viral control in HIV elite controllers and further investigations are warranted 287 to better define the role played by antigen-specific NK cell responses in this rare subset of PLWH.

288 Overall, we addressed two major deficits to the field: we demonstrate that a conserved and 289 epitope-specific targetable mechanism dependent on NKG2C/HLA-E underlies true antigen290 specific memory NK cell responses in humans, and together with previous observations, our 291 detailed phenotypic and transcriptional analyses suggest complex phenotypes may be the most 292 effective at tracking antigen-specific memory NK cells in vivo rather than individual markers. 


\section{MATERIALS AND METHODS}

\section{Generation of functional autologous BLCL}

296 EBV-transformed B cell lines (BLCL) were generated as autologous targets for antigen-specific

297 NK cell assays using standard protocols. Briefly, PBMC for each donor were incubated with EBV 298 supernatant $37^{\circ} \mathrm{C}$ for 1 hour. After washing, PBMC were cultured for 3-6 weeks in R10 299 supplemented with $1 \mathrm{ug} / \mathrm{ml}$ CSA, being split approximately every 7 days. After achieving log 300 phase growth, for which maximum antigen processing and presentation occurs, BLCL were used

301 in subsequent assays. BLCL functional capacity was confirmed by processing of DQ-Ovalbumin 302 DQ assay (Molecular Probes). Briefly, BLCL were incubated with $1 \mathrm{mg} / \mathrm{mL}$ DQ for 2 hours. DQ-O 303 cleavage was confirmed by flow cytometry analysis on an LSRII instrument against negative 304 controls including BLCL without DQ-O and with DQ-O added but kept at 4 degrees.

\section{Flow cytometry-based antigen-specific NK cell killing assay}

307 K562 cells and autologous BLCL were labeled with 1uM CellTrace ${ }^{\mathrm{TM}}$ Violet Cell Proliferation Kit 308 (Invitrogen) for 20 minutes according to manufacturers' instructions. CellTrace Violet-labelled

309 BLCL were then pulsed for 1.5 hours at $37 \mathrm{C}$ with $2 \mathrm{ug} / \mathrm{mL}$ HIV Gag (HIV-1 Consensus B; by the

310 NIH AIDS Reagent Program) or CEF (CMV, EBV and influenza)-derived overlapping peptide

311 pools in culture medium consisting in RPMI-1640 supplemented with 2 mM L-glutamine, 100

$312 \mathrm{ug} / \mathrm{ml}$ streptomycin, $100 \mathrm{U} / \mathrm{ml}$ penicillin and 5\% human serum, washed with PBS and

313 resuspended in culture medium. Non-pulsed BLCL served as internal control and were labeled

314 with 2uM CellTrace ${ }^{\mathrm{TM}}$ CFSE Cell Proliferation Kit (Invitrogen) for 15 minutes according to

315 manufacturers' instructions, washed and resuspended in culture medium. NK cells were purified

316 using AutoMACS NK cell enrichment kit and resuspended in culture medium with $0.1 \mathrm{ng} / \mathrm{mL}$

317 recombinant human IL-15. 350,000-400,000 NK cells were co-cultured with BLCL at 10:1 E:T

318 ratio containing an equal mixture of pulsed target cells and non-pulsed control cells or K562 cells 
319 for 16 hours at $1 \mathrm{M} / \mathrm{mL}$, and subsequently stained using CD3-A700 (UCHT1), CD19-BV711

320 (HIB19), CD16-BV785 (3G8) and CD56-PEcy7 (B159). Specific lysis of BLCL was calculated as

321 follows: (\% sample lysis with NK effectors - \% basal lysis without NK effectors) / (100 - \% basal

322 lysis without NK effectors) (Fig. S2).

323

324 Analysis of primary NK cell or NKCL responses to HIV and influenza peptide antigens

325 by intracellular cytokine staining

326 To measure primary antigen-specific NK cell responses, cryopreserved PBMCs from healthy

327 donors or PLWH were thawed and used either unfractionated, after CD3+ T cell depletion using

328 the EasySep Human CD3 Positive Selection Kit II and the and the protocol for Using EasySep

329 Positive Selection Kits for Cell Depletion provided by the manufacturer (STEMCELL

330 Technologies) or after NK cell enrichment using the EasySep Human NK Cell Enrichment Kit

331 (STEMCELL Technologies). To measure antigen-specific NKCL responses, NKCL were never

332 cryopreserved and used directly from cultures after phenotypic analysis to confirm expression of

333 CD16, CD56 in the absence of CD3. Autologous BLCL were pulsed for 1.5 to 2 hours at $37 \mathrm{C}$ with

$3342 \mathrm{ug} / \mathrm{mL}$ of peptide pools consisting of 15 -mer sequences with 11 aa overlap covering the

335 complete sequence of the influenza virus A/California/04/09(H1N1) NP (PepTivator, Miltenyi),

336 A/California/08/2009(H1N1) MP1 (PepMix, JPT Peptide Technologies), CMV pp65 (NIH AIDS

337 Reagent Program), HIV Env and HIV Gag (both from NIH AIDS Reagent Program) or with 5ug/mL

338 of single nonameric peptides derived from influenza NP, HIV Env, HIV Gag or CMV UL40

339 (Thermofisher). After washing, peptide-loaded BLCL were co-cultured with enriched NK cells or

$340 \mathrm{NKCL}$ at a 1:1 E:T ratio at $37 \mathrm{C}$ for $4 \mathrm{~h}$ to $15 \mathrm{~h}$ with CD107a BV786 or CD107a FITC (H4A3) (BD

341 Biosciences). In some instances $1 \mathrm{ng} / \mathrm{mL}$ of recombinant human IL-15 (R\&D) was also added.

342 Unfractionated PBMC from PLWH or CD3-depleted PBMCs from healthy donors were stimulated

343 directly with peptides without addition of BLCL. 1uL/mL GolgiPlug (BD Biosciences) and 0.7uL/mL

344 GolgiStop (BD Biosciences) were added for the whole $4 \mathrm{~h}$ of incubation or, when incubation lasted 
345 over $4 \mathrm{~h}$, for the last $2 \mathrm{~h}$ of incubation. Cells were stained first with the LIVE/DEAD® Fixable Blue

346 Dead Cell Stain Kit (Invitrogen), then with CD3 BV510 or CD3 A700 (UCHT1), CD14 BV421

347 (M5E2), CD19 BV421 (HIB19), CD16 APC-Cy7 (3G8) and CD56 BV605 (NCAM16.2 or B159) to

348 gate on NK cells, and finally fixed, permeabilized (Thermofisher Fix and Perm) and stained with

349 IFN- $\gamma$ FITC (B27) antibodies to detect intracellular cytokines (all BD Biosciences antibodies). In

350 all assays described above, incubation in the presence of $5 \mu \mathrm{g} / \mathrm{mL}$ of phytohemagglutinin (PHA)

351 or PMA/ionomycin were used as positive controls and unstimulated NK cells or, when applicable,

352 non-pulsed BLCL served as negative controls and for background subtraction. A Fluorescence

353 Minus One (FMO) control and PHA-stimulated PBMCs were used to set the gates for positive

354 cytokine responses. Acquisition of data was performed on a BD LSRII instrument. Data was

355 analyzed using Flow Jo v.10.7.1.

\section{Generation of primary NK cell clones}

358 Single primary human NK cells were cloned by limiting dilution or single-cell sorting using a

359 protocol adapted from a previously reported method ${ }^{62}$ (Fig. S4). Briefly, NK cells were isolated

360 from PBMC via negative selection (EasySep Cell Separation; StemCell Technologies), added to

361 a mix of irradiated feeders consisting of freshly isolated allogeneic PBMC combined with log-

362 phase-growth RPMI 8866 cells (Sigma-Aldrich) at a 10:1 ratio in cloning medium. Cloning

363 medium is composed of RPMI-1640 supplemented with $5 \%$ human serum (Sigma-Aldrich), 1X

364 MEM-NEAA (Gibco), 1X sodium pyruvate (Gibco), $100 \mu \mathrm{g} / \mathrm{mL}$ kanamycin, $300 \mathrm{U} / \mathrm{mL}$ Roche

365 recombinant human IL-2 (Sigma-Aldrich) and $1 \mu \mathrm{g} / \mathrm{mL}$ phytohaemagglutinin (PHA; Fisher). The

366 co-cultures were mixed thoroughly and plated at $100 \mu \mathrm{L} /$ well (1 NK cell/well) in 96-well plates.

367 After 14 days of incubation at $37^{\circ} \mathrm{C} / 5 \% \mathrm{CO} 2$, wells that had outgrowth of cells were transferred to

368 48-well plates and maintained in NK-cell cloning medium with frequent media exchange

369 (approximately every 3 days). NK cell clones were stained with the following antibodies for flow 
370 cytometric phenotyping: BD Biosciences CD3-A700 (UCHT1), CD16-APC-Cy7 (3G8), CD56-

371 BV605 (NCAM16.2), Beckman Coulter NKG2A PE-Cy7 (Z199), and R\&D NKG2C-PE (134591).

372 Only NK cell clones that were CD3-CD56+ were used for subsequent assays.

373

374 Human Subjects

375 De-identified and coded blood samples from the majority of HIV-negative healthy donors used in

376 this study were collected under IRB-approved protocols and delivered to us by Research Blood

377 Components LLC (Watertown, Massachusetts). Additional healthy donors and all PLWH were

378 recruited from outpatient clinics at Massachusetts General Hospital and affiliated Boston-area

379 hospitals or at UAB University Hospital. The respective institutional review boards approved

380 this study, and all subjects gave written informed consent. A total of 4 elite controllers, 27

381 untreated viremic chronic progressors, and 20 c-ART-treated chronically infected PLWH were

382 studied (Table S1). Elite controllers were defined as having plasma HIV RNA levels of $<50$

383 copies/ml in the absence of antiretroviral therapy, on at least three determinations over at

384 least a year of follow-up. Viremic controllers had detectable HIV-1 RNA levels of $<2,000$

385 copies/ml. Untreated viremic chronic progressors were defined as subjects having untreated

386 HIV infection for $>1$ year with plasma viral loads of $>2,000$ copies $/ \mathrm{ml}$ for at least 1 year of

387 follow-up; cART treated chronically infected had HIV RNA levels below the limit of detection

388 for the respective available standard assays (e.g., <75 RNA copies/ml by branched DNA

389 assay or $<50$ copies by PCR). PBMCs were isolated from whole blood by Ficoll - Hypaque

390 density gradient centrifugation, frozen (90\% FBS - 10\% DMSO), and either used immediately or

391 stored in LN2 vapor until analyzed.

392

393 Calcein acetoxymethyl (AM)-based antigen-specific NK cell killing assay for NKCL 
394 Autologous BLCL were stained with $10 \mu \mathrm{m}$ Calcein AM (Invitrogen) for $1 \mathrm{~h}$, then washed three

395 times prior to be pulsed with overlapping peptide pools encompassing HIV-1 Consensus B Gag

396 or Env (NIH AIDS Reagent Program), influenza A/California/04/2009(H1N1) NP (PepTivator,

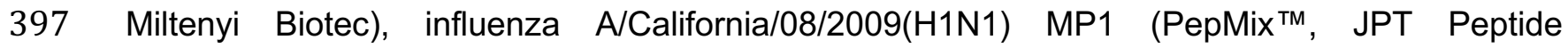

398 Technologies), CMV pp65 (NIH AIDS Reagent Program), and Myelin-oligodendrocyte

399 glycoprotein (MOG) negative control (PepMix ${ }^{\mathrm{TM}}$, JPT Peptide Technologies) or with individual

400 nonameric peptides. Peptide-pulsed BLCL or mock-pulsed controls were then incubated with or

401 without NK cell clones at a 5:1 E:T ratio. Supernatant were harvested after $4 \mathrm{~h}$ incubation at

$40237^{\circ} \mathrm{C} / 5 \% \mathrm{CO} 2$. Release of CAM into the supernatant was measured using a fluorescence reader

403 (excitation $485 \mathrm{~nm}$, absorption $530 \mathrm{~nm}$ ). The percent-specific lysis was calculated as follows: (test

404 release - spontaneous release)/(maximum release - spontaneous release) $\times 100$.

406 RNA Isolation

407 Between 100,000 and 500,000 NKCL were pelleted (500 x g, for 10 minutes) and dry pellets

408 immediately frozen at $-80^{\circ} \mathrm{C}$. Pellets were lysed by vortexing for 1 minute in $350 \mathrm{uL}$ cold 409 supplemented RLT buffer (RLT $+\beta-\mathrm{MeOH})$ at $4^{\circ} \mathrm{C}$ and lysates homogenized using QIAshredder

410 columns (Qiagen). RNA was then extracted from these samples using the RNeasy Plus Micro kit

411 (Qiagen) with on-column DNase digestion. After isolation of total RNA the RNA integrity was

412 analyzed with the RNA 6000 Pico Chip on an Agilent 2100 Bioanalyzer (Agilent Technologies).

\section{RNA transcriptome analysis}

415 Prior to library generation, RNA was subjected to DNAse I digestion (Thermo Fisher Scientific)

416 followed by RNeasy MinElute column clean up (Qiagen). RNA-Seq libraries were generated using

417 the SMART-Seq v4 Ultra Low Input RNA Kit (Clontech Laboratories) as per the manufacturer's 418 recommendations. From cDNA final libraries were generated utilizing the Nextera XT DNA Library 
419 Preparation Kit (Illumina). Concentrations of the final libraries were measured with a Qubit 2.0

420 Fluorometer (Thermo Fisher Scientific) and fragment lengths distribution was analyzed with the

421 DNA High Sensitivity Chip on an Agilent 2100 Bioanalyzer (Agilent Technologies). All samples

422 were normalized to $2 \mathrm{nM}$ and pooled equimolar. The library pool was sequenced on the

423 NextSeq500 (Illumina) with 1x75bp, with 14 to 19 mio reads per sample.

424 Transcript expression was quantified with salmon v. 0.14.0 using default parameters against

425 the GRCh38 human genome reference. Differential gene expression (DGE) was performed using

426 DESeq2 v. 1.28.1 with a previously described protocol ${ }^{63,64}$. An adjusted p-value cutoff of 0.05 was

427 used to determine significance. Differentially regulated genes were selected by calculation of a

428 false-discovery rate below 0.05 and log2 fold change values greater than or equal to 1.5 and less

429 than or equal to -1.5 . Gene symbols and Entrez identifiers were mapped to Ensembl gene

430 identifiers using biomaRt v. $2.44 .1 \mathrm{~d}^{65,66}$. Gene set enrichment analysis (GSEA) was used with

431 Entrez IDs and ranked based on the following formula:

432

433

$$
-\log _{10}(\text { adjusted } p \text {-value }) * \operatorname{sign}\left(\log _{2} \text { Fold Change }\right)
$$

Msigdbr v. 7.1.1 was used to retrieve Gene Ontology (GO) and Hallmark pathways ${ }^{67}$ and GSEA

435 was performed using the ranked gene list and pathways with the function fgseaSimple() from the

436 fgsea v. 1.14.0 package with default parameters ${ }^{68}$. Heatmaps were generated using pheatmap v.

$4371.0 .12^{69}$. Volcano plots and GSEA results were generated using ggplot2 v. 3.3.2 ${ }^{70}$.

439 Advanced Polychromatic flow cytometry for the phenotypic characterization of NKCL

440 NKCL previously frozen in liquid nitrogen were stained with panel of antibodies detailed in Table

441 S2. Data were acquired on a FACS Symphony A5 flow cytometer (BD Biosciences) equipped with

442 five lasers (UV, 355 nm; violet, 405 nm; blue, 488; yellow/green, $561 \mathrm{~nm}$; red, $637 \mathrm{~nm}$; all tuned

443 at $100 \mathrm{~mW}$, except UV tuned at $60 \mathrm{~mW}$ ). Flow Cytometry Standard (FCS) files were exported as

444 FCS3.0 from DiVa 8.0.1. FCS files were loaded and compensated in FlowJo by using single - 
445 stained controls (Compbeads incubated with fluorescently conjugated antibodies). After

446 compensation and gating, live NKCL were exported as FCS3.0 files for subsequent t-SNE

447 analysis using CytoDRAV (https://github.com/ReevesLab/CytoDRAV).

449 HLA-E stabilization assay

450 A selection of nonameric peptides derived from subtype B HIV Gag (60) (GenBank accession

451 number ABR15476.1) and Env (84) (UniProtKB accession number A0A160I6K4) as well as

452 influenza A/California/04/2009(H1N1) NP (23) (UniProtKB accession number C3W5S2) predicted

453 to have strong and weak binding to HLA-E*01:01 by the NetMHCpan 4.0 and Immune Epitope

454 Database servers were synthesized using the PEPotec Immuno Custom Peptide Libraries

455 service provided by Thermofisher Scientific (Table S3). Analyses were conducted using influenza

456 NP, HIV Gag and HIV Env sequences matching those used to generate the peptide pools used

457 in our studies. The ability of individual peptides to stabilize surface expression of human HLA-E

458 was assessed using K562-HLA-E 01:01 transfectants cultured at $26^{\circ} \mathrm{C}$ for $26 \mathrm{~h}$ prior to be pulsed

459 with $10 \mu \mathrm{g} / \mathrm{mL}$ of individual peptides for $16 \mathrm{~h}$ at $26^{\circ} \mathrm{C}$. Cell surface expression levels of HLA-E were

460 assessed by flow cytometry staining using anti-HLA-E-BV421 antibody (3D12). HLA-E expression

461 was compared to that upon pulsing with positive control peptide VL9 (VMAPRTLIL), a canonical

462 CMV UL40/HLA-Cw3 leader sequence-derived peptide that stabilizes HLA-E, and negative

463 control peptide NLVPMVATV, a CMV pp65-derived nonamer that does not stabilize HLA-E. Data

464 were acquired on an LSRII instrument (BD Biosciences), and analyzed using FlowJo software

465 v10.7.1 (Treestar).

466

467 Acknowledgements

468 We thank all the patients who contributed to this study making this work possible. 


\section{$470 \quad$ Funding}

471 This research was supported by National Institutes of Health $(\mathrm{NIH})$ grants: R01Al116363,

472 R21Al137835 (to S.J.) and R01Al120828, R01Al143457, UM1 Al124377 (to R.K.R.). We also

473 acknowledge support from the CVVR Flow Cytometry and Harvard University Center for AIDS

474 Research Advanced Laboratory Technologies Core (P30 Al060354).

\section{Author Contributions}

477 S.J. and R.K.R. designed the study, analyzed data and generated the final figures. O.L., T.Y., 478 S.S., S.S., R.J., G.T., A.W., P.J.T., H.L.D. and J.G. performed the experiments and analyzed 479 data. A.G. performed the RNAseq with input from M.A. K.K. performed the bioinformatic analyses. 480 P.G. and M.A. provided access to human samples. M.M.T. provided crucial reagents. S.J. and 481 R.K.R. wrote the manuscript with contributions of all authors.

\section{Competing Interests}

484 The authors declare no competing financial interests.

\section{Figure Legends}

487 Fig. 1 | Human NK cells mediate antigen-specific responses against HIV and influenza.

488 a, Enriched NK cells from PLWH or healthy donors were co-cultured with autologous BLCL that 489 had been pulsed with $2 \mathrm{ug} / \mathrm{mL}$ peptide pools derived from HIV Gag (HIV-1 Consensus B; provided 490 by the NIH AIDS Reagent Program) and NK cell responses assessed by ICS. Dead cells were 491 excluded. Dot plots show proportions of IFN-y+ and CD107a+ NK cells after subtracting 492 background (unstimulated). Asterisks indicate significant differences between healthy donors and 493 samples from PLWH. b, Autologous BLCL were pulsed with a pool of HIV Gag overlapping 494 peptides or with the CEF (CMV, EBV and influenza) control peptide pool and were labeled with 
495 the CellTrace Violet dye. Mock-pulsed BLCL serving as intra-well controls were labeled with the 496 green dye CFSE. Purified NK cells were co-cultured with BLCL at 5:1 E:T ratios (equal mixture of 497 pulsed target BLCL and non-pulsed control BLCL) for 16 hours, and specific lysis of BLCL was 498 determined by flow cytometry. Killing of HLA-deficient K562 cells was used as additional positive 499 control. c, Enriched NK cells 11 HIV-negative healthy donors were incubated overnight with $5002 \mathrm{ug} / \mathrm{mL}$ peptide pools derived from influenza A/California/04/2009(H1N1) NP and

501 A/California/08/2009(H1N1) MP1 or CMV pp65 and NK cell responses assessed by ICS. Dead

502 cells were excluded. Dot plots show proportions of IFN-y+ and CD107a+ NK cells after subtracting

503 background (unstimulated). Asterisks, significant differences compared to unstimulated controls. $504 * p<0.05$

505

506 Fig. 2 | Single-cell cloning of human antigen-specific NK cells. Percentages of antigen507 specific lysis by a, 46 (out of 159) NKCL from 20 PLWH that reacted against HIV and b, 19 (out 508 of 40) NKCL from 8 healthy donors that reacted against influenza. CAM cytotoxicity assays were 509 used to evaluate lysis after co-culture of NKCL with autologous BLCL pulsed with indicated 510 peptide pools. Non-specific lysis was assessed by measuring killing of mock-pulsed autologous

511 BLCL. NKCL reactive against HIV antigens were identified as NKCL mediating specific killing at

512 least twice above specific killing of self-peptides-pulsed B-LCL after background subtraction, and,

513 for those with no self-peptide available data, at least twice above non-specific killing (mock pulsed

514 B-LCL). NKCL found unreactive or over-reactive based on those criteria are not displayed.

516 Fig. 3 | Human antigen-specific NK cell responses are associated with NKG2C expression.

517 a, Hierarchical clustering and heatmap of the 16 differentially expressed genes distinguishing 518 HIV-specific NKCL $(n=8)$ and NKCL not reacting to HIV Gag/Env $(n=7)$ from the same untreated 519 viremic PLWH. Normalized Z-scores are displayed as a colorimetric scale of blue (lower 520 expression) to red (higher expression). b, Bar graphs show mean + SEM proportions of NKCL 
521 expressing $\alpha 4 \beta 7$, CCR5 and KLRG1 evaluated by flow cytometry and comparing 5 NKCL from 2

522 healthy donors (HD) with 14 NKCL from one untreated viremic PLWH, including 7 HIV Gag/Env-

523 specific NKCL and 7 NKCL that did not react to HIV Gag/Env. c, Non-linear t-SNE (bh-SNE) plots

524 of 14 NKCL from one untreated viremic PLWH, showing distinct clustering for 7 HIV reactive and

5257 non HIV-reactive NKCL, with HIV-reactive NKCL being partly associated with cell clusters

526 expressing high levels of the inhibitory NKG2A or high levels of activating NKG2C and/or inhibitory

527 KIR3DL1. d, Antigen-specific killing of HIV Env-pulsed BLCL by 6 NKCL in the presence of isotype

528 control or NKG2C blocking antibodies. Bars represent mean+SEM. e, Spearman correlation

529 analysis between frequencies of NKG2C+ NKCL and specific killing of BLCL pulsed with influenza

530 NP, MP1 or HA.

531

532

Fig. 4 | Human antigen-specific memory NK cell responses are mediated via recognition of

533 HLA-E-binding viral peptides. a, K562 cells stably expressing HLA-E*0101 were either left

534 mock pulsed or pulsed for $16 \mathrm{~h}$ with $10 \mathrm{ug} / \mathrm{mL}$ of nonameric peptides derived from HIV consensus

535 B Gag (60 total) or Env (84 total) or from A/California/04/2009(H1N1) NP (23 total). Controls

536 included CMV pp65-derived NLVPMVATV that do not stabilize HLA-E (NEG), and VMAPRTLIL,

537 a CMV/HLA-Cw3 leader sequence-derived peptide that stabilizes HLA-E (POS). HLA-E surface

538 stabilization was assessed by flow cytometry. Bars represent mean percentages of HLA-E+ K562

539 cells + SEM for indicated individual peptides or from all non-stabilizing peptides $(n)$ pooled from

540 at least 3 distinct experiments. Dot plots represent percentages of antigen- or peptide-specific

541 lysis by 17 NKCL from 5 untreated viremic PLWH (b) and 20 NKCL from 5 healthy donors (c)

542 after subtracting non-specific lysis (killing of mock pulsed autologous BLCL). CAM cytotoxicity

543 assays were used to evaluate lysis after co-culture of NKCL with autologous BLCL pulsed with

544 peptide pools encompassing the whole HIV Env, HIV Gag or H1N1 NP sequence or with indicated

545 single HLA-E-binding nonameric peptides derived from CMV UL40, HIV Gag, HIV Env, or H1N1 
546 NP. Asterisks, significant differences compared to mock-pulsed pulsed prior to background

547 subtraction. ${ }^{*} p<0.05,{ }^{* *} p<0.01$.

548

549 Fig. 5 | HIV Env- and H1N1 NP-derived HLA-E-binding peptides elicit primary NK cell

550 responses. a, PBMCs samples from 15 PLWH (11 cART-treated and 4 untreated viremic) were

551 incubated for $16 \mathrm{~h}$ with $2 \mu \mathrm{g} / \mathrm{mL}$ of 1 pool of 15 amino acid peptides overlapping by 11 amino acids

552 and spanning HIV Env or single HIV Env- and CMV UL40-derived nonamers in the presence of

553 CD107a antibodies. GolgiStop and GolgiPlug were added for the last $2 \mathrm{~h}$ incubation prior to

554 surface and intracellular cytokine staining. b, Purified NK cells from 9 healthy donors were

555 incubated for $4 \mathrm{~h}$ with autologous BLCL pulsed with $2 \mu \mathrm{g} / \mathrm{mL}$ of 1 pool of 15 amino acid peptides

556 overlapping by 11 amino acids and spanning H1N1 NP or single H1N1 NP- and CMV UL40-

557 derived nonamers in the presence of CD107a antibodies, GolgiStop and GolgiPlug. Dot plots

558 represent proportions of IFN-y producing (left panel) or actively degranulating, cytotoxic NK cells,

559 as judged by cell surface expression of CD107a (right panel) after subtracting proportions of IFN-

$560 y+$ and CD107a+ unstimulated NK cells, respectively. Bars represent the median.

561 


\section{References}

5631 Florez-Alvarez, L., Hernandez, J. C. \& Zapata, W. NK Cells in HIV-1 Infection: From

564 Basic Science to Vaccine Strategies. Front Immunol 9, 2290,

565 doi:10.3389/fimmu.2018.02290 (2018).

5662 Scully, E. \& Alter, G. NK Cells in HIV Disease. Current HIVIAIDS reports 13, 85-94,

567 doi:10.1007/s11904-016-0310-3 (2016).

5683 Jost, S. \& Altfeld, M. Control of Human Viral Infections by Natural Killer Cells. Annual

569 review of immunology, doi:10.1146/annurev-immunol-032712-100001 (2013).

5704 Shah, S. V. et al. CMV Primes Functional Alternative Signaling in Adaptive Deltag NK

$571 \quad$ Cells but Is Subverted by Lentivirus Infection in Rhesus Macaques. Cell Rep 25, 2766-

$572 \quad 2774$ e2763, doi:1016/j.celrep.2018.11.020 (2018).

5735 O'Leary, J. G., Goodarzi, M., Drayton, D. L. \& von Andrian, U. H. T cell- and B cell-

$574 \quad$ independent adaptive immunity mediated by natural killer cells. Nat Immunol 7, 507-516

575 (2006).

5766 Sun, J. C., Beilke, J. N. \& Lanier, L. L. Adaptive immune features of natural killer cells.

$577 \quad$ Nature 457, 557-561, doi:10.1038/nature07665 (2009).

5787 Paust, S. et al. Critical role for the chemokine receptor CXCR6 in NK cell-mediated

579 antigen-specific memory of haptens and viruses. Nat Immunol 11, 1127-1135,

580 doi:10.1038/ni.1953 (2010).

5818 Gillard, G. O. et al. Thy1+ NK [corrected] cells from vaccinia virus-primed mice confer

582 protection against vaccinia virus challenge in the absence of adaptive lymphocytes.

$583 \quad$ PLoS pathogens 7, e1002141, doi:10.1371/journal.ppat.1002141 (2011).

5849 Foley, B. et al. Cytomegalovirus reactivation after allogeneic transplantation promotes a $585 \quad$ lasting increase in educated NKG2C+ natural killer cells with potent function. Blood 119, 586 2665-2674, doi:10.1182/blood-2011-10-386995 (2012).

58710 Peng, H. et al. Liver-resident NK cells confer adaptive immunity in skin-contact

588 inflammation. The Journal of clinical investigation 123, 1444-1456,

589 doi:10.1172/JCl66381 (2013).

59011 Lee, J. et al. Epigenetic modification and antibody-dependent expansion of memory-like

$591 \quad$ NK cells in human cytomegalovirus-infected individuals. Immunity 42, 431-442,

592 doi:10.1016/j.immuni.2015.02.013 (2015).

59312 Paust, S., Blish, C. A. \& Reeves, R. K. Redefining Memory: Building the Case for

594 Adaptive NK Cells. Journal of virology 91, doi:10.1128/JVI.00169-17 (2017). 
59513 Reeves, R. K. et al. Antigen-specific NK cell memory in rhesus macaques. Nature

596 immunology 16, 927-932, doi:10.1038/ni.3227 (2015).

$59714 \mathrm{Li}, \mathrm{T}$. et al. Respiratory Influenza Virus Infection Induces Memory-like Liver NK Cells in

598 Mice. Journal of immunology 198, 1242-1252, doi:10.4049/jimmunol.1502186 (2017).

59915 Hammer, Q. et al. Peptide-specific recognition of human cytomegalovirus strains

600 controls adaptive natural killer cells. Nat Immunol 19, 453-463, doi:10.1038/s41590-018-

601 0082-6 (2018).

$60216 \quad$ Gardiner, C. M. \& Mills, K. H. The cells that mediate innate immune memory and their

603 functional significance in inflammatory and infectious diseases. Semin Immunol 28, 343-

$604 \quad 350$, doi:10.1016/j.smim.2016.03.001 (2016).

60517 Blok, B. A., Arts, R. J., van Crevel, R., Benn, C. S. \& Netea, M. G. Trained innate

606 immunity as underlying mechanism for the long-term, nonspecific effects of vaccines. $J$

607 Leukoc Biol 98, 347-356, doi:10.1189/jlb.5RI0315-096R (2015).

60818 Nikzad, R. et al. Human natural killer cells mediate adaptive immunity to viral antigens.

609 Sci Immunol 4, doi:10.1126/sciimmunol.aat8116 (2019).

61019 Wijaya, R. S. et al. HBV vaccination and HBV infection induces HBV-specific natural

$611 \quad$ killer cell memory. Gut, doi:gutjnl-2019-319252 (2020).

61220 Stary, V. et al. A discrete subset of epigenetically primed human NK cells mediates

613 antigen-specific immune responses. Sci Immunol 5, doi:10.1126/sciimmunol.aba6232

614 (2020).

61521 Ma, M. et al. NKG2C(+)NKG2A(-) Natural Killer Cells are Associated with a Lower Viral

616 Set Point and may Predict Disease Progression in Individuals with Primary HIV Infection.

617 Front Immunol 8, 1176, doi:10.3389/fimmu.2017.01176 (2017).

61822 Gondois-Rey, F. et al. NKG2C(+) memory-like NK cells contribute to the control of HIV

619 viremia during primary infection: Optiprim-ANRS 147. Clin Transl Immunology 6, e150,

620 doi:10.1038/cti.2017.22 (2017).

62123 Thomas, R. et al. NKG2C deletion is a risk factor of HIV infection. AIDS Res Hum

622 Retroviruses 28, 844-851, doi:10.1089/AID.2011.0253 (2012).

62324 Peppa, D. et al. Adaptive Reconfiguration of Natural Killer Cells in HIV-1 Infection. Front

$624 \quad$ Immunol 9, 474, doi:10.3389/fimmu.2018.00474 (2018).

62525 Suliman, S. et al. Bacillus Calmette-Guerin (BCG) Revaccination of Adults with Latent

626 Mycobacterium tuberculosis Infection Induces Long-Lived BCG-Reactive NK Cell

627 Responses. Journal of immunology 197, 1100-1110, doi:10.4049/jimmunol.1501996

628 (2016). 
62926 Rolle, A., Meyer, M., Calderazzo, S., Jager, D. \& Momburg, F. Distinct HLA-E Peptide 630 Complexes Modify Antibody-Driven Effector Functions of Adaptive NK Cells. Cell Rep 24, 1967-1976 e1964, doi:10.1016/j.celrep.2018.07.069 (2018).

63227 Pereyra, F. et al. Genetic and immunologic heterogeneity among persons who control 633 HIV infection in the absence of therapy. The Journal of infectious diseases 197, 563571, doi:10.1086/526786 (2008).

63528 Guma, M. et al. Imprint of human cytomegalovirus infection on the NK cell receptor 636 repertoire. Blood 104, 3664-3671 (2004).

63729 Hendricks, D. W. et al. Cutting edge: NKG2C(hi)CD57+ NK cells respond specifically to acute infection with cytomegalovirus and not Epstein-Barr virus. Journal of immunology 192, 4492-4496, doi:10.4049/jimmunol.1303211 (2014). subset during acute human cytomegalovirus infection. Proceedings of the National Academy of Sciences of the United States of America 108, 14725-14732, doi:10.1073/pnas.1110900108 (2011).

64732 Tomasec, P. et al. Surface expression of HLA-E, an inhibitor of natural killer cells, enhanced by human cytomegalovirus gpUL40. Science 287, 1031, doi:science.287.5455.1031 (2000).

65033 Pietra, G. et al. HLA-E-restricted recognition of cytomegalovirus-derived peptides by human CD8+ cytolytic T lymphocytes. Proc Natl Acad Sci U S A 100, 10896-10901, doi:pnas.1834449100 (2003).

$65334 \quad$ Nattermann, J. et al. The HLA-A2 restricted T cell epitope HCV core 35-44 stabilizes HLA-E expression and inhibits cytolysis mediated by natural killer cells. Am J Pathol 166, 443-453, doi:10.1016/S0002-9440(10)62267-5 (2005).

65635 Garcia, P. et al. Human T cell receptor-mediated recognition of HLA-E. Eur J Immunol 657 32, 936-944, doi:10.1002/1521-4141(200204)32:4<936::AID-IMMU936>3.0.CO;2-M 658 (2002).

$65936 \quad$ Nattermann, J. et al. HIV-1 infection leads to increased HLA-E expression resulting in 660 impaired function of natural killer cells. Antivir Ther 10, 95-107 (2005). 
66137 Davis, Z. B. et al. A Conserved HIV-1-Derived Peptide Presented by HLA-E Renders 662 Infected T-cells Highly Susceptible to Attack by NKG2A/CD94-Bearing Natural Killer 663 Cells. PLoS pathogens 12, e1005421, doi:10.1371/journal.ppat.1005421 (2016).

66438 Hannoun, Z. et al. Identification of novel HIV-1-derived HLA-E-binding peptides.

665 Immunol Lett 202, 65-72, doi:10.1016/j.imlet.2018.08.005 (2018).

66639 Aktas, E., Erten, G., Kucuksezer, U. C. \& Deniz, G. Natural killer cells: versatile roles in 667 autoimmune and infectious diseases. Expert Rev Clin Immunol 5, 405-420, 668 doi:10.1586/eci.09.27 (2009).

66940 Alter, G., Malenfant, J. M. \& Altfeld, M. CD107a as a functional marker for the identification of natural killer cell activity. J Immunol Methods 294, 15-22, doi:10.1016/j.jim.2004.08.008 (2004). Hansen, S. G. et al. Profound early control of highly pathogenic SIV by an effector memory T-cell vaccine. Nature 473, 523-527, doi:10.1038/nature10003 (2011). Hansen, S. G. et al. A live-attenuated RhCMVISIV vaccine shows long-term efficacy against heterologous SIV challenge. Sci Transl Med 11, doi:10.1126/scitransImed.aaw2607 (2019). Rhesus Macaques, and Cynomolgus Macaques. J Immunol 200, 49-60, doi:10.4049/jimmunol.1700841 (2018). Adaptive NK Cells. J Virol 91, doi:10.1128/JVI.00169-17 (2017). of nonselective and specific NK cell activation. J Immunol 190, 6269-6276, doi:10.4049/jimmunol.1202533 (2013). Venkatasubramanian, S. et al. IL-21-dependent expansion of memory-like NK cells enhances protective immune responses against Mycobacterium tuberculosis. Mucosal Immunol 10, 1031-1042, doi:10.1038/mi.2016.105 (2017). virology 84, 8959-8963, doi:10.1128/JVI.01126-10 (2010).

69349 Khakoo, S. I. et al. HLA and NK cell inhibitory receptor genes in resolving hepatitis C virus infection. Science 305, 872-874, doi:10.1126/science.1097670 (2004). 
69550 Malnati, M. S. et al. Activating Killer Immunoglobulin Receptors and HLA-C: a successful

696 combination providing HIV-1 control. Sci Rep 7, 42470, doi:10.1038/srep42470 (2017).

69751 Martin, M. P. et al. Epistatic interaction between KIR3DS1 and HLA-B delays the

698 progression to AIDS. Nat Genet 31, 429-434 (2002).

69952 Martin, M. P. et al. Innate partnership of HLA-B and KIR3DL1 subtypes against HIV-1.

700

701 Nature genetics 39, 733-740, doi:10.1038/ng2035 (2007).

702

53 Fadda, L. et al. Common HIV-1 peptide variants mediate differential binding of KIR3DL1

703 to HLA-Bw4 molecules. Journal of virology 85, 5970-5974, doi:10.1128/JVI.00412-11

70454 Peruzzi, M., Parker, K. C., Long, E. O. \& Malnati, M. S. Peptide sequence requirements for the recognition of HLA-B ${ }^{\star} 2705$ by specific natural killer cells. J Immunol 157, 33503356 (1996). immunodominant viral peptides complexed to HLA-B*2705. Eur J Immunol 35, 341-351, doi:10.1002/eji.200425724 (2005). dependent upon molecular microarchitecture within the Bw4 epitope. J Immunol 194, 781-789, doi:10.4049/jimmunol.1402542 (2015). defining allotypic dimorphism that impacts both HLA and peptide sensitivity. J Immunol 192, 2875-2884, doi:10.4049/jimmunol.1303142 (2014).

Jiang, C. et al. Distinct viral reservoirs in individuals with spontaneous control of HIV-1. Nature 585, 261-267, doi:10.1038/s41586-020-2651-8 (2020). against HIV-1. Nat Immunol 16, 563-570, doi:10.1038/ni.3161 (2015). HIV proteome. Science 364, 480-484, doi:10.1126/science.aav5095 (2019).

61 Migueles, S. A. et al. Lytic granule loading of CD8+ T cells is required for HIV-infected cell elimination associated with immune control. Immunity 29, 1009-1021, doi:10.1016/j.immuni.2008.10.010 (2008).

Cella, M. \& Colonna, M. Cloning human natural killer cells. Methods in molecular biology 121, 1-4, doi:10.1385/1-59259-044-6:1 (2000). 
72763 Love, M. I., Huber, W. \& Anders, S. Moderated estimation of fold change and dispersion

728 for RNA-seq data with DESeq2. Genome Biol 15, 550, doi:10.1186/s13059-014-0550-8

729 (2014).

73064 Love, M. I., Huber, W. \& Anders, S. Analyzing RNA-seq data with DESeq2. Retrieved

731 from

732 https://bioconductor.org/packages/release/bioc/vignettes/DESeq2/inst/doc/DESeq2.html.

733 (2020).

73465 Durinck, S. et al. BioMart and Bioconductor: a powerful link between biological

735 databases and microarray data analysis. Bioinformatics 21, 3439-3440,

736 doi:10.1093/bioinformatics/bti525 (2005).

73766 Durinck, S., Spellman, P. T., Birney, E. \& Huber, W. Mapping identifiers for the

738 integration of genomic datasets with the R/Bioconductor package biomaRt. Nat Protoc 4,

739 1184-1191, doi:10.1038/nprot.2009.97 (2009).

74067 Dolgalev, I. msigdbr: MSigDB Gene Sets for Multiple Organisms in a Tidy Data Format

741 (Version 7.1.1). Retrieved from https://CRAN.R-project.org/package=msigdbr. (2020).

74268 Korotkevich, G., Sukhov, V. \& Sergushichev, A. Fast gene set enrichment analysis.

743 BioRxiv. Preprint published online October 22, 2019, doi:https://doi.org/10.1101/060012

744 (2019).

74569 Kolde, R. pheatmap: Pretty Heatmaps (Version 1.0.12). Retrieved from https://CRAN.R-

746 project.org/package=pheatmap. (2019).

74770 Wickham, H. ggplot2: Elegant graphics for data analysis (Second edition). Springer.

748 (2016).




\section{Supplem entary Table 1. Characteristics of PLWH and healthy donors}

\begin{tabular}{lcccc|}
\hline Clinical Data & $\begin{array}{c}\text { Virem ic } \\
(\mathbf{n = 2 7})\end{array}$ & $\begin{array}{c}\text { cART treated } \\
(\mathbf{n}=\mathbf{2 0})\end{array}$ & $\begin{array}{c}\text { elite controllers } \\
(\mathbf{n = 4})\end{array}$ & $\begin{array}{c}\text { HIV-negative } \\
(\mathbf{n = 3 9 )}\end{array}$ \\
\hline Median HIV viral load [IQR] (RNA cop/mL) & $14,100[28,790]$ & $<50$ & $<50$ & NA \\
\hline Median CD4 cell count [IQR] (cells/mm3) & $599[286]$ & $496.5[355]$ & & NA \\
N with available clinical data & 26 & 2 & 0 & NA \\
\hline
\end{tabular}

PLWH, People living with HIV; cART, combination antiretroviral therapy; IQR, interquartile range; NA, not applicable 750

\begin{tabular}{|c|c|c|c|}
\hline \multicolumn{4}{|c|}{ Supplementary Table 2. 28-color panel for NKCL phenotyping } \\
\hline Target & Fluorochrome & Clone & Manufacturer \\
\hline LIVE cells & Blue Live/Dead & & Invitrogen \\
\hline CD19 & PE-CY5.5 & J3-119 & BECKMAN COULTER \\
\hline CD14 & BUV615 & M5E2 & BD Pharmingen \\
\hline CD3 & BUV496 & UCHT1 & BD Pharmingen \\
\hline CD4 & BB700 & L200 & BD Pharmingen \\
\hline CD56 & BUV737 & NCAM16.2 & BD Pharmingen \\
\hline CD16 & BUV563 & $3 G 8$ & BD Pharmingen \\
\hline NKP46 & BV711 & 9E2/NKp46 & BD Pharmingen \\
\hline NKG2D & BB790 & $1 \mathrm{D} 11$ & BD Pharmingen \\
\hline NKG2C & Biotin + SA BUV395 & REA205 & MILTENYI \\
\hline NKG2A & PE-CY7 & Z199 & BECKMAN COULTER \\
\hline KIR3DL1/S1 & VioBlue & REA168 & MILTENYI \\
\hline KIR2DL1/S1/S3/S5 & FITC & HP-MA4 & BIOLEGEND \\
\hline KIR2DS4 & APC-Vio770 & REA284 & MILTENYI \\
\hline CD85J & $\mathrm{PE}$ & $\mathrm{GHI} / 75$ & BD Pharmingen \\
\hline$\alpha 4 \beta 7$ & APC & & NHP Reagent Resource \\
\hline CXCR6 & BV786 & 13B 1E5 & BD Pharmingen \\
\hline CCR5 & PE-CY5 & 2D7/CCR5 & BD Pharmingen \\
\hline CCR7 & APC-R700 & 3D12 & BD Pharmingen \\
\hline 2B4 & BV650 & $2-69$ & BD Pharmingen \\
\hline CD2 & BV510 & $2 \mathrm{H} 7$ & BD Pharmingen \\
\hline CD8 & BV570 & RPA-T8 & BIOLEGEND \\
\hline CD57 & BB630 & NK-1 & BD Pharmingen \\
\hline KLRG1 & PE-Dazzle594 & 2F1/KLRG1 & BIOLEGEND \\
\hline PD1 & BV605 & $\mathrm{EH} 12.1$ & BD Pharmingen \\
\hline TIM-3 & BV750 & 7D3 & BD Pharmingen \\
\hline HLA-DR & BUV661 & G46-6 & BD Pharmingen \\
\hline IL-7R & BUV805 & HIL-7R-M21 & BD Pharmingen \\
\hline
\end{tabular}


bioRxiv preprint doi: https://doi.org/10.1101/2020.11.09.374348; this version posted November 10, 2020. The copyright holder for this preprint (which was not certified by peer review) is the author/funder. All rights reserved. No reuse allowed without permission.

\begin{tabular}{|c|c|c|c|c|c|c|c|c|}
\hline \multicolumn{3}{|c|}{ HIV Gag } & \multicolumn{3}{|c|}{ HIV Env } & \multicolumn{3}{|c|}{ A/California/7/2009(H1N1) NP } \\
\hline ID & Sequence & Position & ID & Sequence & Position & ID & Sequence & Position \\
\hline 1 & VWASRELER & 35 & 36 & LWRWGTMLL & 13 & 1 & TMDSNTLEL & 373 \\
\hline 2 & SRELERFAV & 38 & 37 & TMLLGMLMI & 18 & 2 & CLPACVYGL & 275 \\
\hline 3 & ERFAVNPGL & 42 & 38 & VWKEATTTL & 43 & 3 & VQRNLPFER & 414 \\
\hline 4 & ILGQLQPSL & 60 & 39 & EATTTLFCA & 46 & 5 & IQMCTELKL & 41 \\
\hline 5 & QVSQNYPIV & 127 & 40 & ATHACVPTD & 69 & 28 & RMVLSAFDE & 65 \\
\hline 6 & QNYPIVQNL & 130 & 41 & ENVTENFNM & 86 & 29 & ACHSAAFED & 332 \\
\hline 7 & VHQAISPRT & 143 & 42 & QMHEDIISL & 102 & 30 & VASGHDFER & 285 \\
\hline 8 & VVEEKAFSP & 158 & 43 & IISLWDQSL & 107 & 11B & LRGSVAHKS & 266 \\
\hline 9 & FSPEVIPMF & 164 & 44 & VQKEYALFY & 168 & 15B & FLARSALIL & 258 \\
\hline 10 & PEVIPMFSA & 166 & 45 & QKEYALFYK & 169 & $16 \mathrm{~B}$ & LMQGSTLPR & 166 \\
\hline 11 & QMLKETINE & 199 & 46 & VITQACPKV & 200 & 17B & VIPRGKLST & 352 \\
\hline 12 & HAGPIAPGQ & 219 & 47 & ACPKVSFEP & 204 & 18B & GMDPRMCSL & 158 \\
\hline 13 & WMTNNPPIP & 249 & 48 & VSFEPIPIH & 208 & 19B & MMESAKPED & 447 \\
\hline 14 & TNNPPIPVG & 251 & 49 & CAPAGFAIL & 218 & $70 \mathrm{C}$ & ATYQRTRAL & 146 \\
\hline 15 & IVRMYSPTS & 273 & 50 & KCNDKKFNG & 227 & $71 \mathrm{C}$ & ATNPIVPSF & 471 \\
\hline 16 & RMYSPTSIL & 275 & 51 & IRPVVSTQL & 251 & $72 \mathrm{C}$ & RLIQNSITI & 55 \\
\hline 17 & YSPTSILDI & 277 & 52 & VIRSENFTD & 271 & $73 \mathrm{C}$ & RMIGGIGRF & 31 \\
\hline 18 & RQGPKEPFR & 286 & 53 & IGPGRAFYT & 309 & $74 \mathrm{C}$ & MSNEGSYFF & 481 \\
\hline 19 & QGPKEPFRD & 287 & 54 & GNKTIVFNQ & 353 & $75 \mathrm{C}$ & ASNENVETM & 366 \\
\hline 20 & RDYVDRFYK & 294 & 55 & CNTTQLFNS & 383 & $76 \mathrm{C}$ & ATAGLTHIM & 129 \\
\hline 21 & VKNWMTETL & 313 & 56 & TKDKNTITL & 402 & $77 \mathrm{C}$ & KLSTRGVQI & 357 \\
\hline 22 & ILKALGPAA & 333 & 57 & TLPCRIKQI & 409 & $78 \mathrm{C}$ & VGIDPFKLL & 299 \\
\hline 23 & LGPAATLEE & 337 & 58 & NNDTEIFRP & 456 & $79 \mathrm{C}$ & WMACHSAAF & 343 \\
\hline
\end{tabular}




\section{SUPPLEMENTARY FIGURE LEGENDS}

754

755

756 Representative flow cytometry plots depicting CellTrace Violet-stained HIV Gag peptide-pulsed

757 and CFSE-stained unpulsed BLCL from a cART-treated PLWH (upper panels), an elite controller

758 (EC) (middle panels) and a healthy donor (lower panels) alone (left panels) or in the presence of

759 autologous purified NK cells (right panels), after gating on CD3neg CD19pos lymphocytes.

760 Representative example of specific lysis calculation is provided based on the following formula:

761 (\% sample lysis with NK effectors - \% basal lysis without NK effectors) / (100 - \% basal lysis

762 without NK effectors).

763

\section{Supplementary Fig. 2}

765 a, Graphical design of the methodology to generate NKCL and evaluate their cytotoxic potential 766 against autologous BLCL presenting viral antigens. b, Representative flow cytometry plots 767 showing CD16 and CD56 expression of three different NKCL generated from PBMC of one single 768 individual.

\section{Supplementary Fig. 3}

771 a, Volcano plot depicting 15 differentially expressed genes distinguishing HIV-specific NKCL

$772(n=8)$ and NKCL not reacting to HIV Gag/Env $(n=7)$ from the same untreated viremic PLWH. Red

773 and blue dots represent significantly upregulated and downregulated genes, respectively.

774 Differentially regulated genes were selected by calculation of a false-discovery rate below 0.05

775 and $\log 2$ fold change values greater than or equal to 1.5 and less than or equal to -1.5 . b, Selected

776 top 10 significant Hallmark pathways for differentially expressed genes in HIV-specific NKCL

777 compared to NKCL not reacting to HIV Gag/Env, as evaluated by Gene Set Enrichment Analysis

778 software using the fgsea v. 1.14.0 package with pathways selected using Msigdbr v. 7.1.1 
779 package. Blue shading indicates an adjusted p-value less than the 0.05 cutoff. Red shading

780 indicates an adjusted $p$-value greater than 0.05 .

\section{Supplementary Fig. 4}

783 Bar graphs show mean + SEM proportions of NKCL expressing indicated surface markers

784 evaluated by flow cytometry and comparing 5 NKCL from 2 healthy donors (HD, white bars) with

78514 NKCL from one untreated viremic PLWH (darker grey bars), including 7 HIV Gag/Env-specific

786 NKCL (dark grey bars) and 7 NKCL that did not react to HIV Gag/Env (light grey bars).

\section{Supplementary Fig. 5}

789 Multidimensional data analysis of $14 \mathrm{NKCL}$ from one untreated viremic PLWH. Live NKCL were

790 analyzed by $t$-SNE with bh-SNE to generate plots clustering cells with similar expression profiles.

791 Relative expression of indicated NK cell markers visualized over the bh-SNE plots is displayed

792 as a colorimetric scale of blue (low expression) to red (high expression).

\section{Supplementary Fig. 6}

795 a, Antigen-specific killing of HIV Env-pulsed BLCL by NKCL in the presence of control or isotype

796 control, NKp30, NKp44 or NKG2C blocking antibodies. b, Percentages of NKG2C+ NKCL,

797 comparing NKCL with more $(>10 \%)$ or less $(<10 \%)$ than $10 \%$ influenza-specific killing for 41

798 NKCL from 9 healthy donors. For each NKCL, the highest antigen-specific killing against influenza

799 HA, NP and MP1 was considered. Bar indicates the median.

800

\section{Supplementary Fig. 7}

802 a, Representative flow cytometry plots showing HLA-E expression on K562 stably expressing

803 HLA-E*01:01 cells that were either left unpulsed (upper panel) or pulsed for $16 \mathrm{~h}$ at $26^{\circ} \mathrm{C}$ with

$8045 \mathrm{ug} / \mathrm{mL}$ of CMV-derived NLVPMVATV that do not stabilize HLA-E (middle panel), or with 
805 VMAPRTLIL, a CMV/HLA-Cw3 leader sequence-derived peptide that stabilizes HLA-E (lower

806 panel). Bar graphs represent mean percentages +SEM of HLA-E+ K562 cells pooled from at least

8072 distinct experiments after pulsing with NLVPMVATV (NEG CTRL), VMAPRTLIL (POS CTRL

808 CMV), 60 single nonameric peptides derived from HIV Gag (b), 84 single nonameric peptides

809 derived from HIV Env (c), or 23 single peptides derived from NP A/California/04/2009(pH1N1) (d)

810 with strong binding prediction scores based on the NetMHC pan 4.0 and IEDB epitope prediction

811 tools. HLA-E surface stabilization was assessed using anti-HLA-E antibody (3D12). Dead cells

812 were excluded. The dotted line marks the cut off for increased HLA-E expression, defined as the

813 mean value plus 2 standard deviations of non-stabilizing peptides.

814

815 
Figure 1.

a

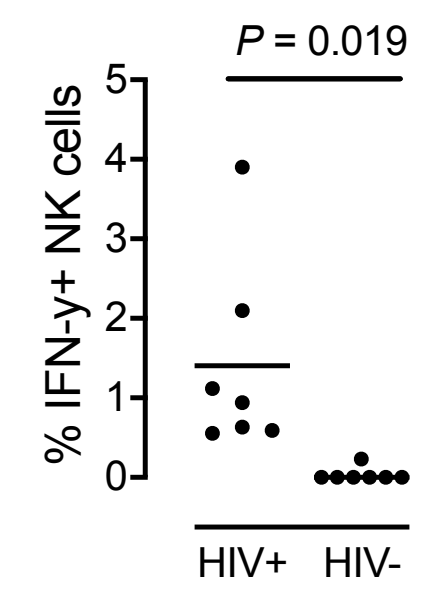

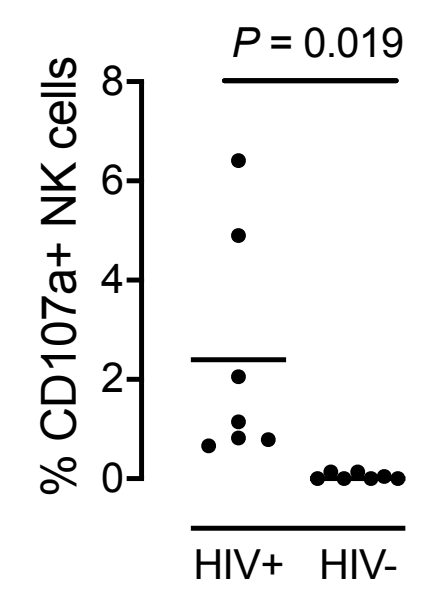

b Elite controllers

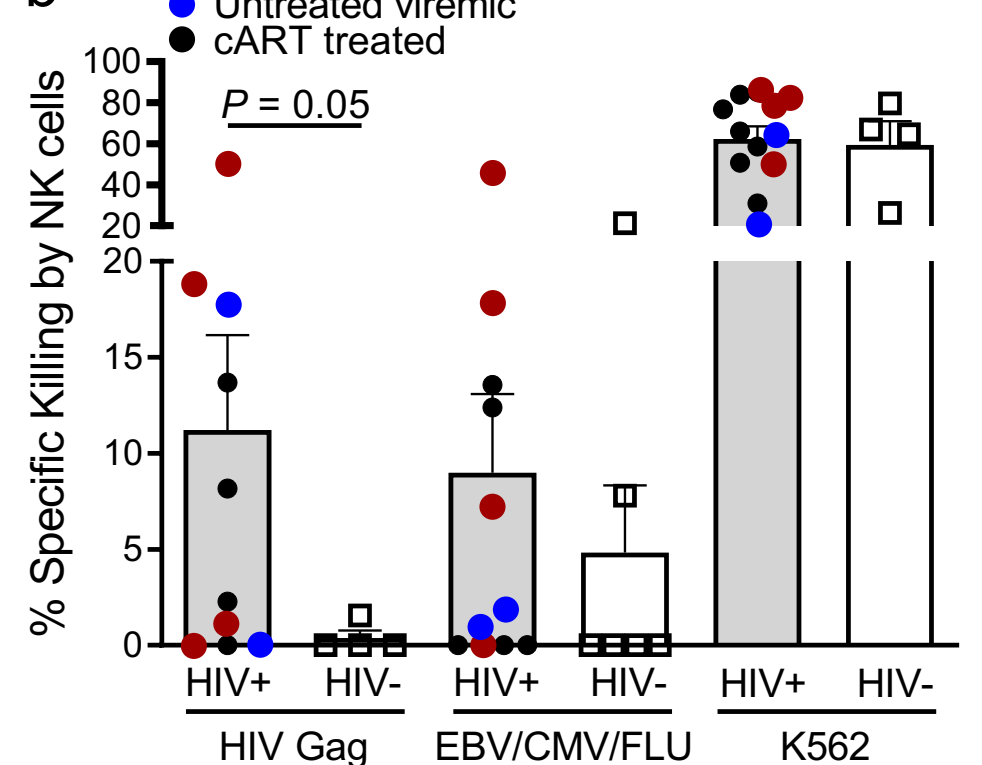

C

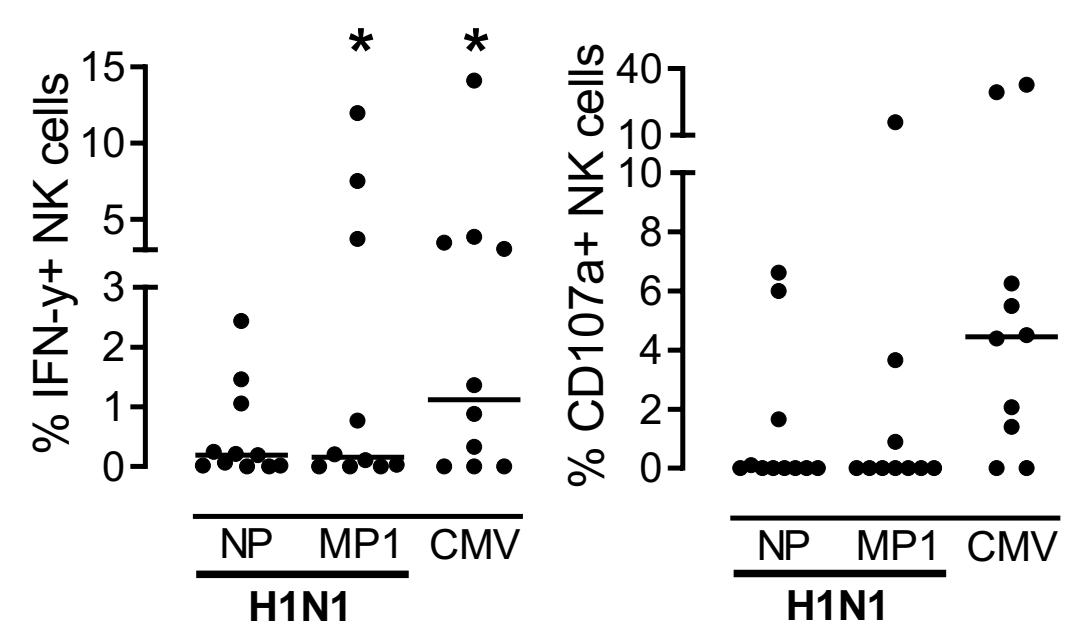


Figure 2.

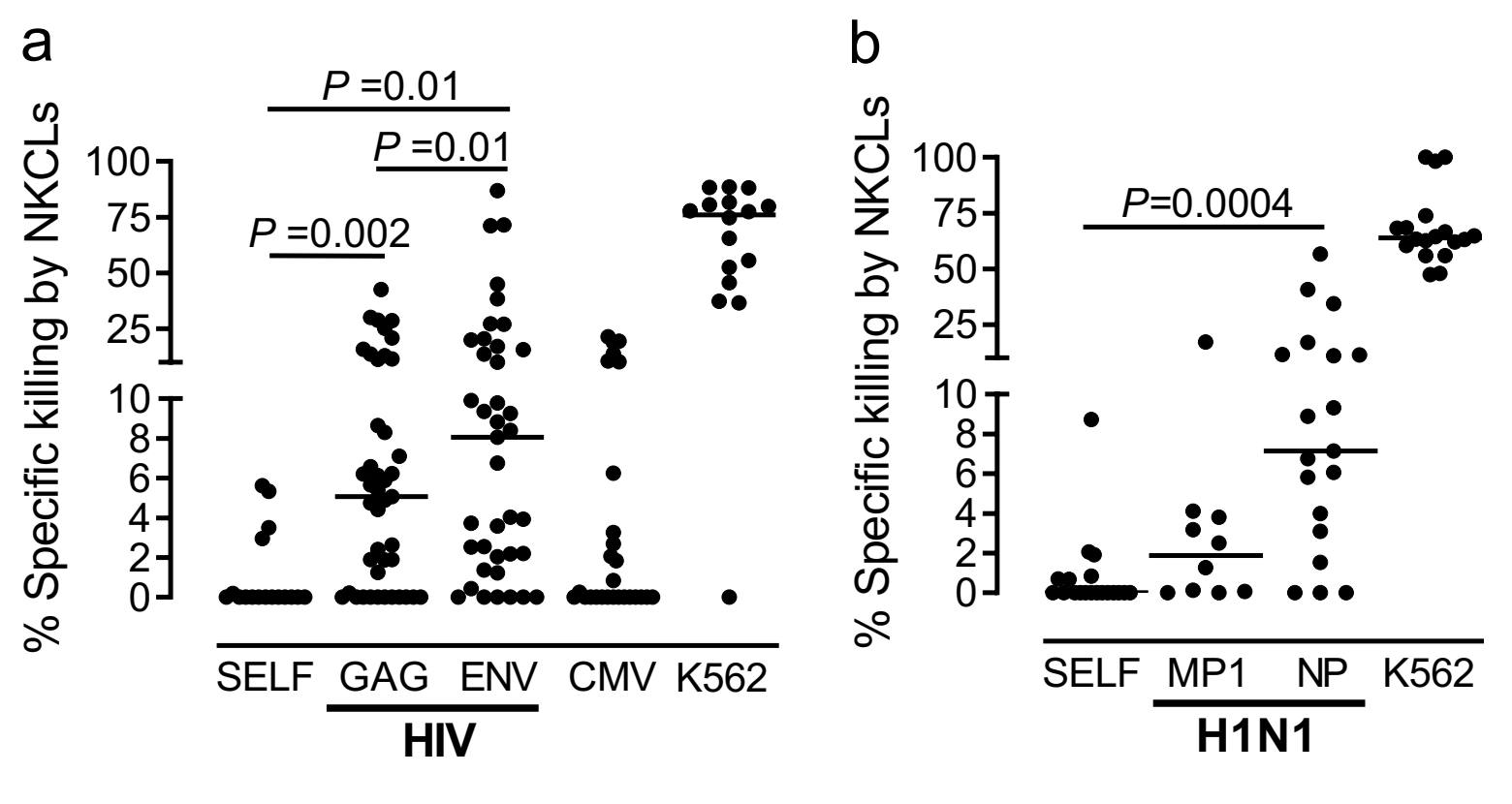

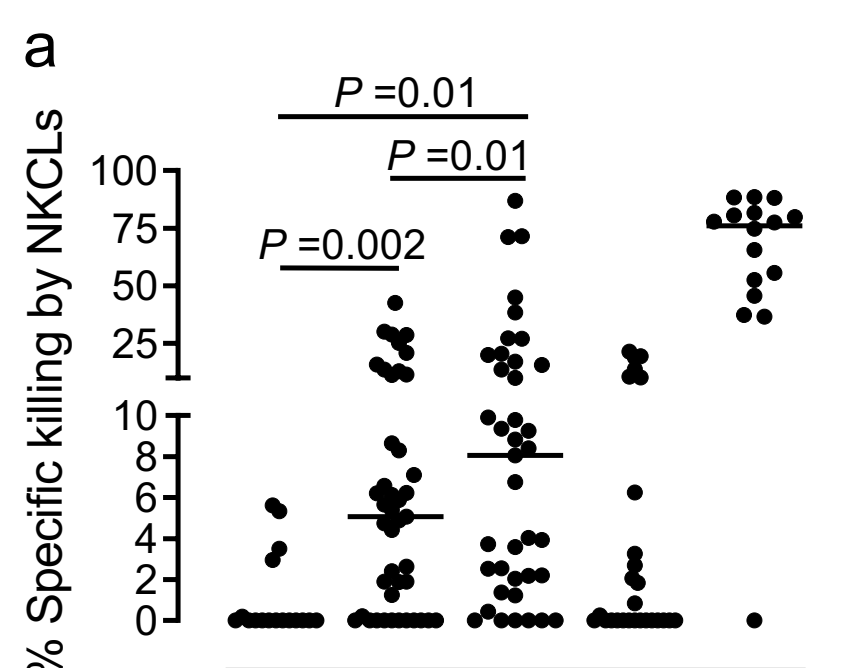

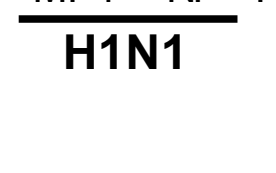


Figure 4
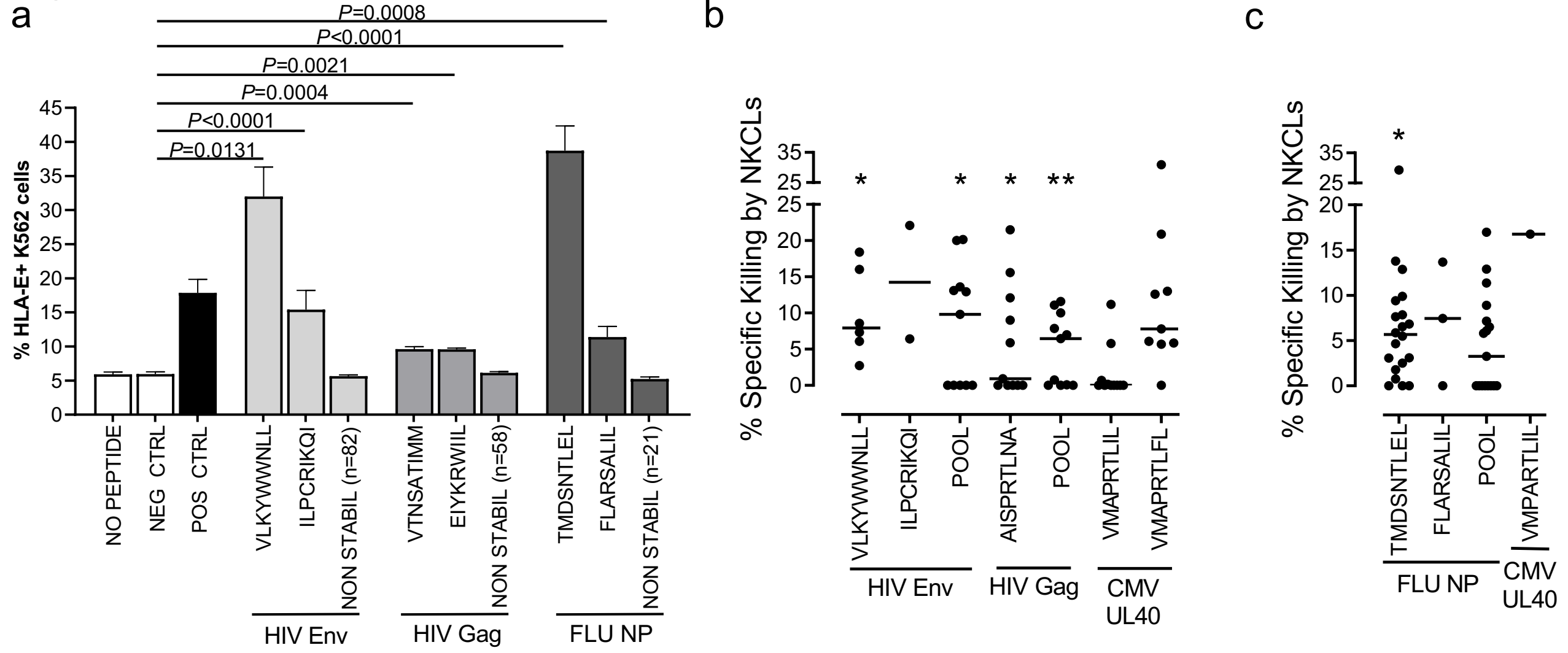
Figure 5.

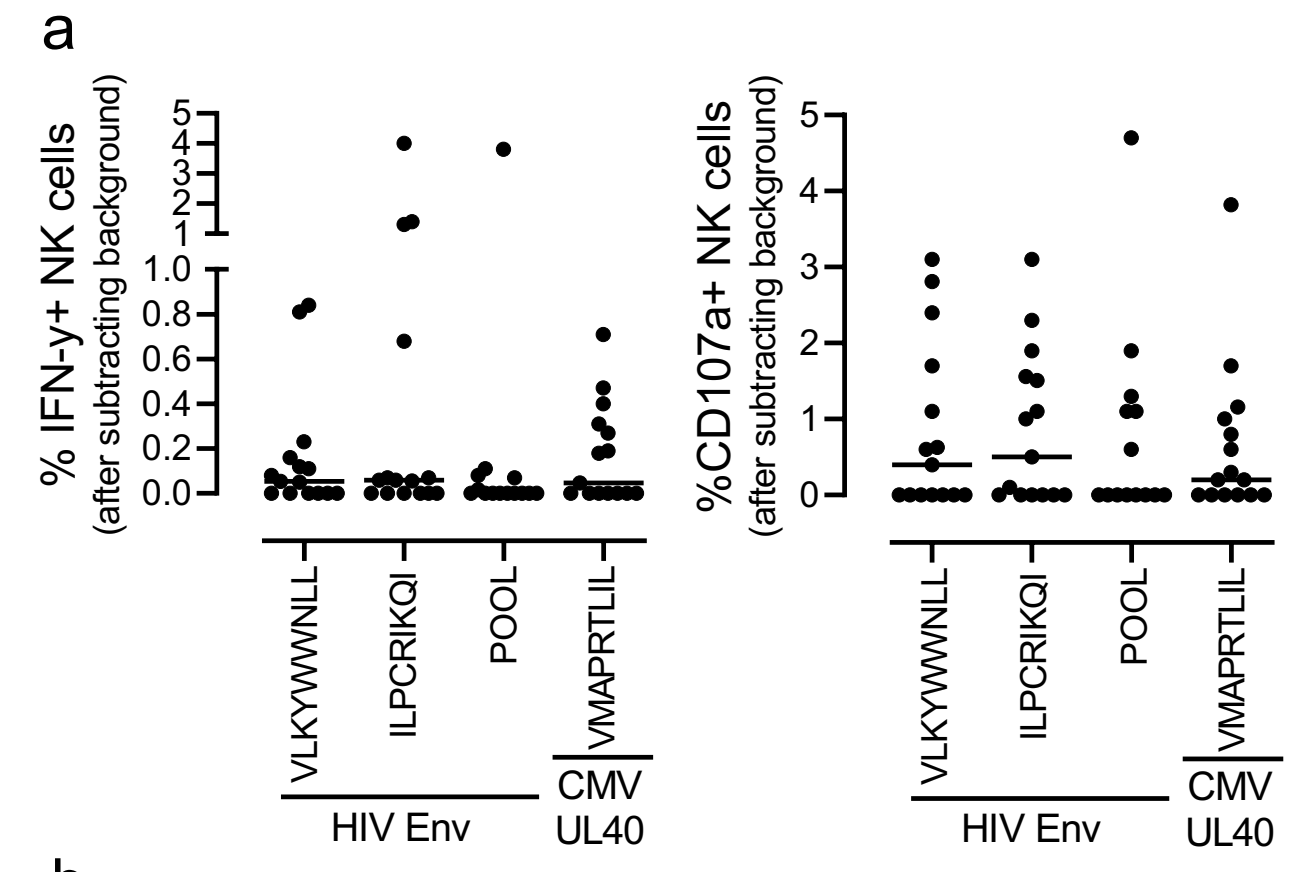

b
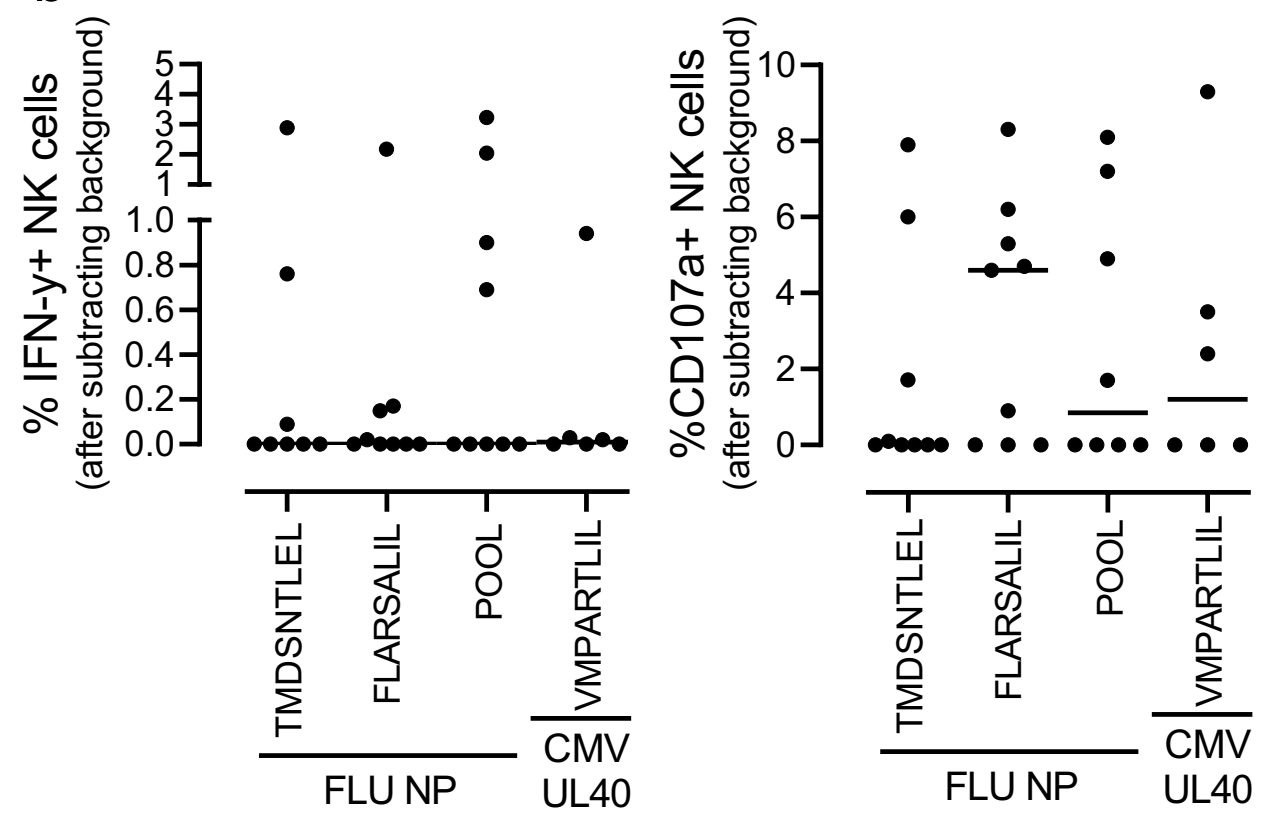
PLWH (CART)

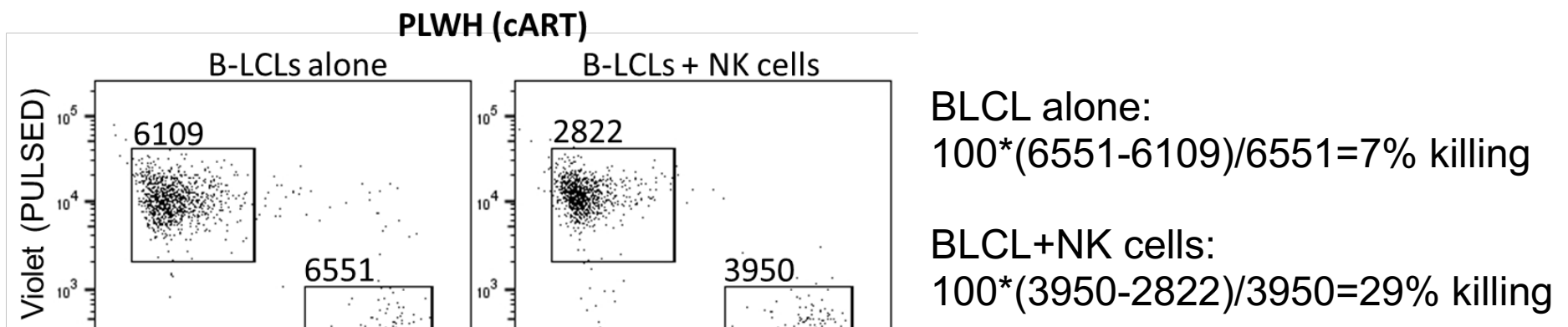

Specific killing:

$(100 *(29 \%-7 \%)) /(100-7 \%)=23 \%$

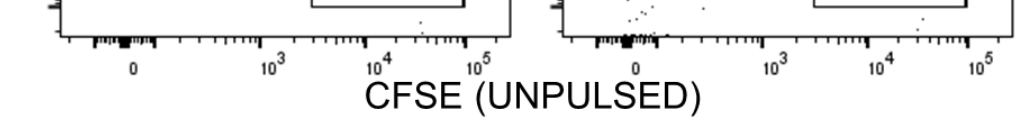

(100* $(29 \%-7 \%)) /(100-7 \%)=23 \%$

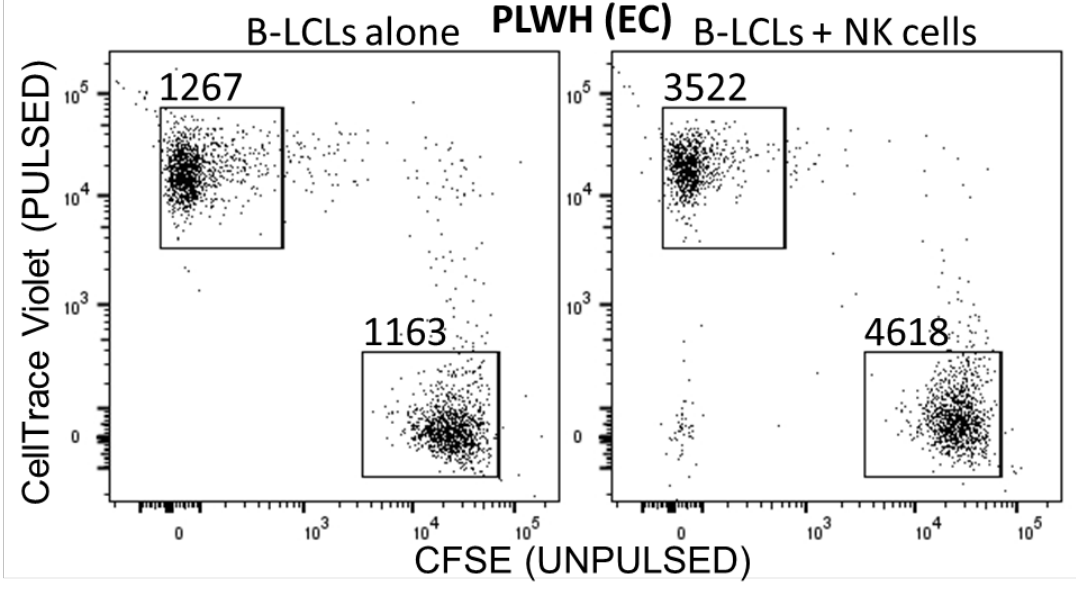

Healthy Donor

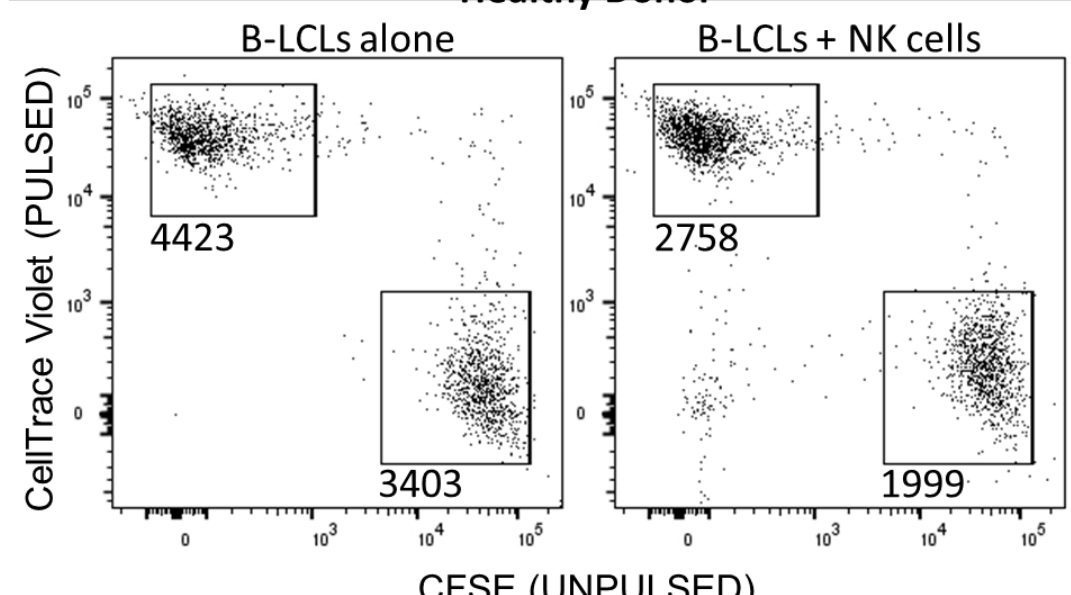

CFSE (UNPULSED) 


\section{Supplementary Fig. 2}
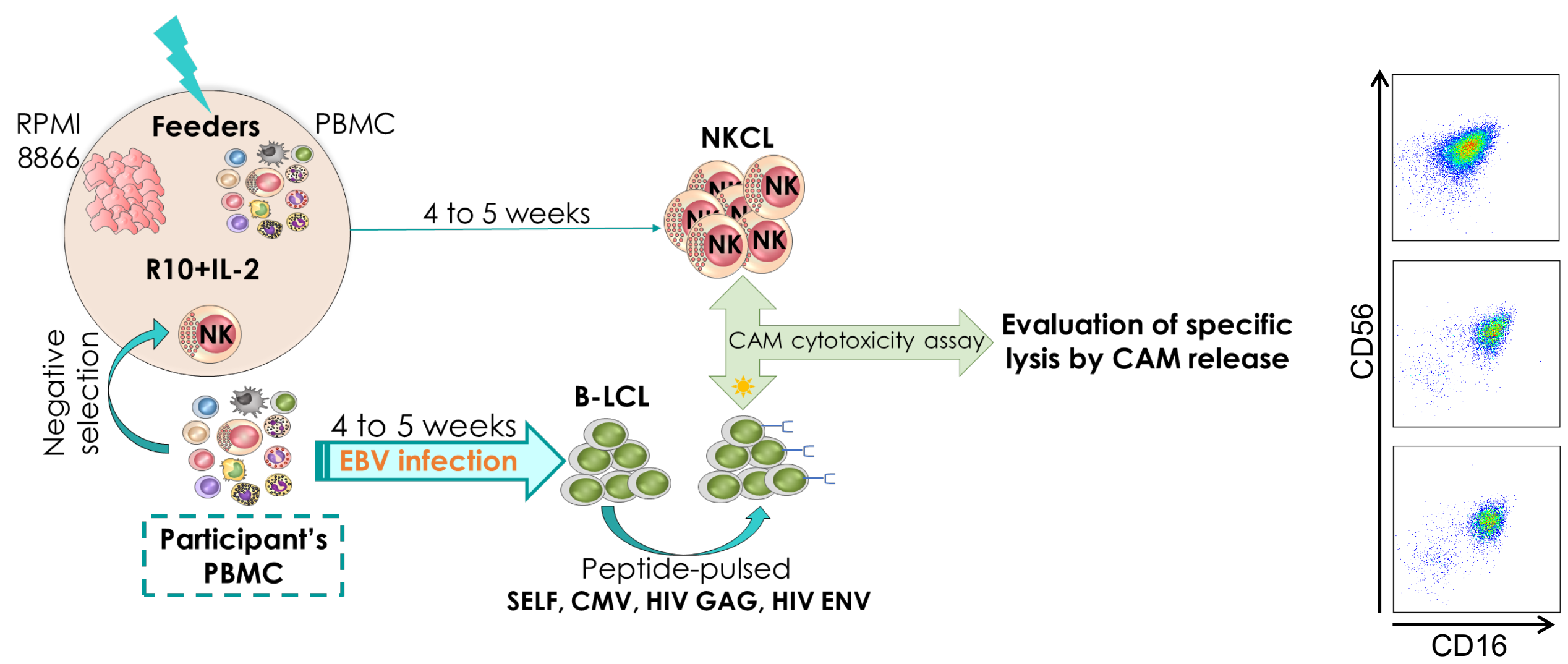
a

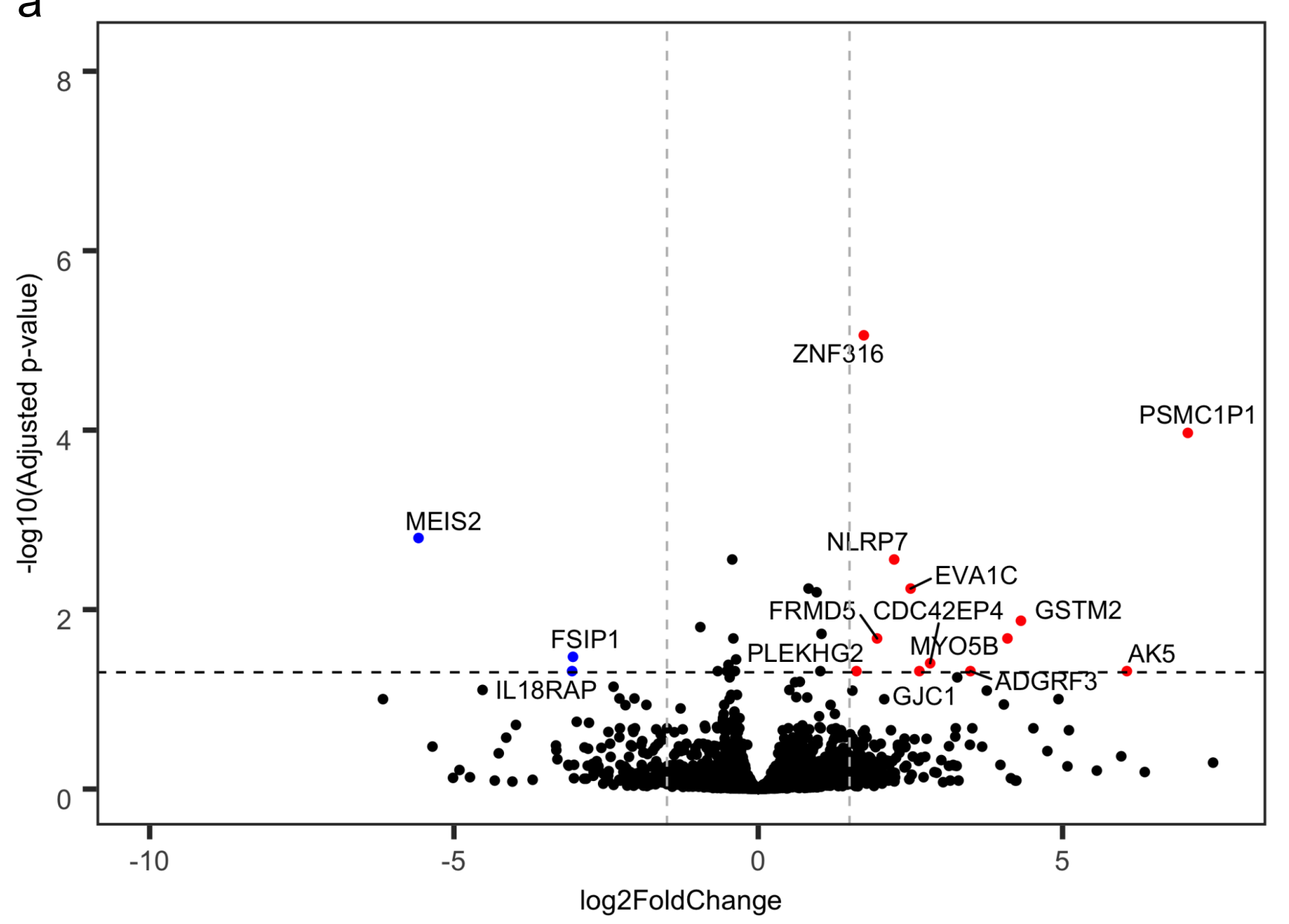

b

MITOTIC SPINDLE

XENOBIOTIC METABOLISM

IFN- $\alpha$ RESPONSE

UNFOLDED PROTEIN RESPONSE

PROTEIN SECRETION

GLYCOLYSIS

ADIPOGENESIS

MYC TARGETS V1

MTORC1 SIGNALING

OXIDATIVE PHOSPHORYLATION

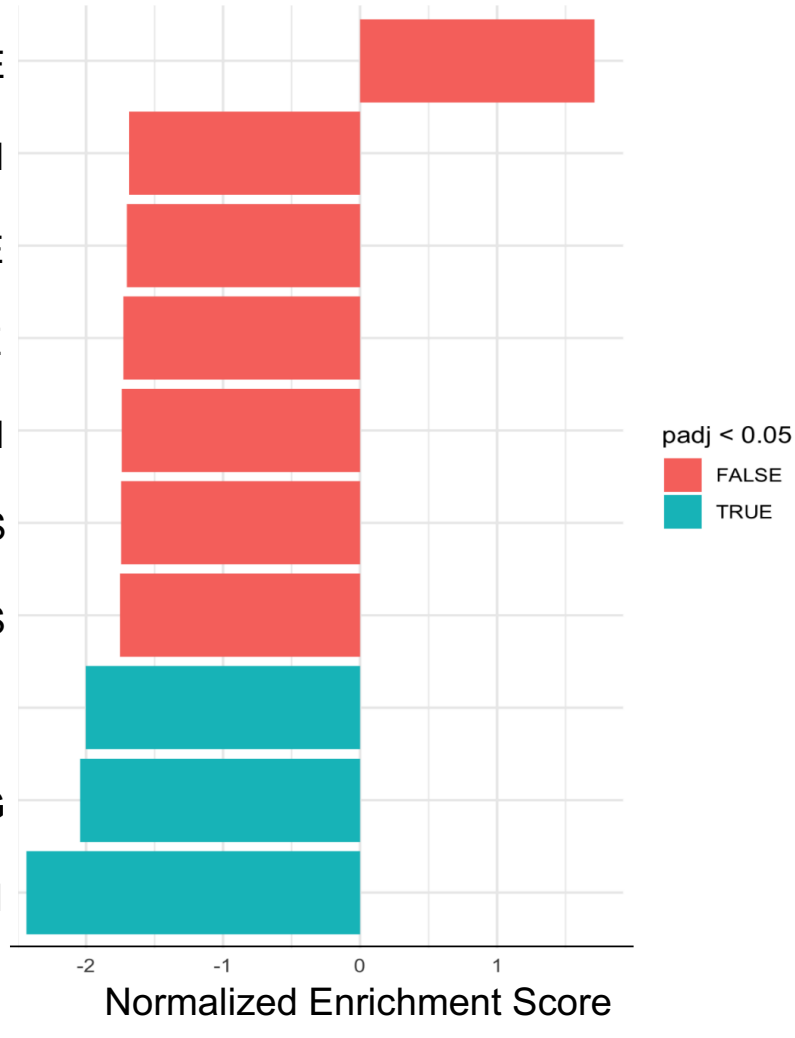




\section{$\square \mathrm{HD}$ \\ PLWH (all)}

$\square$ PLWH (\#1, Non specific to HIV Gag/Env)

PLWH (\#1 HIV Gag/Env-specific)
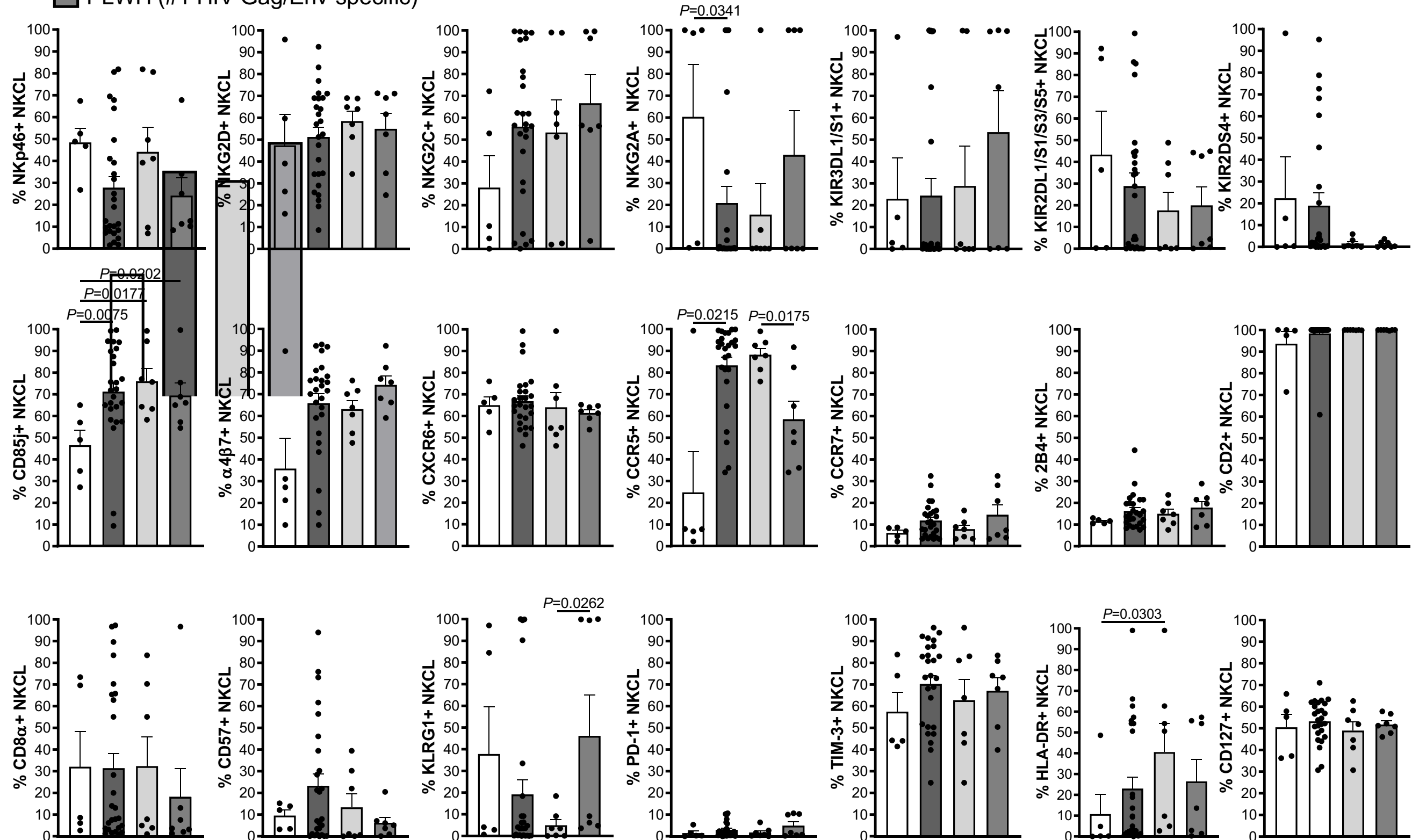
NKG2C

HIV-specific

Non specific to HIV
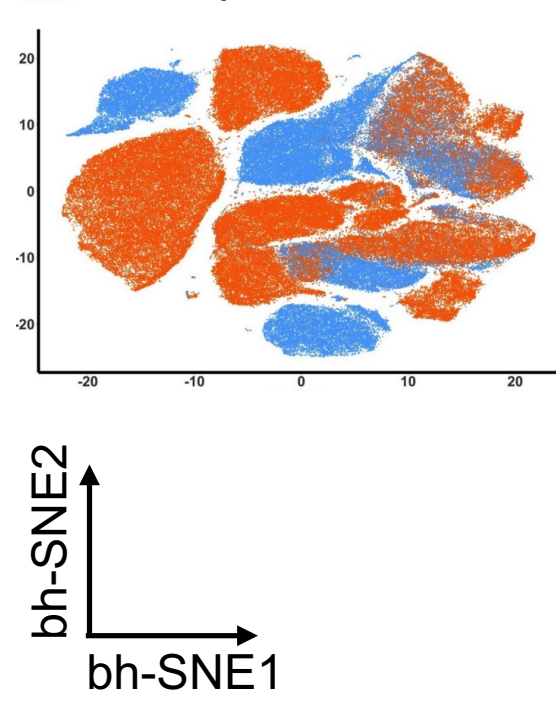

NKG2A
CD16

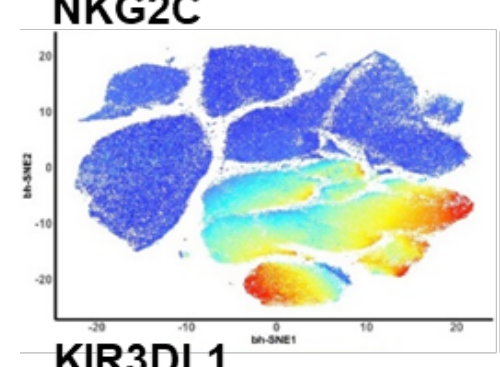

KIR3DL1
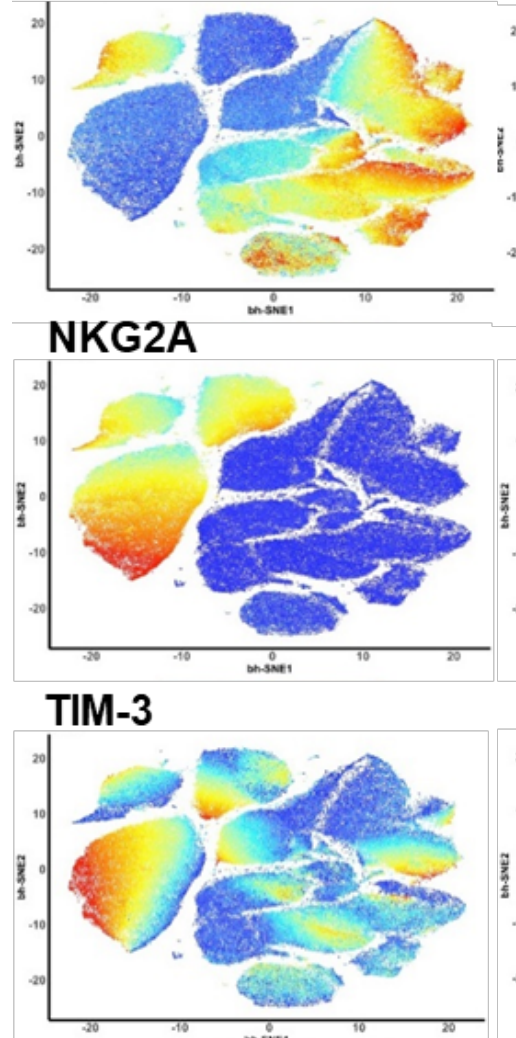

Low

PD-1

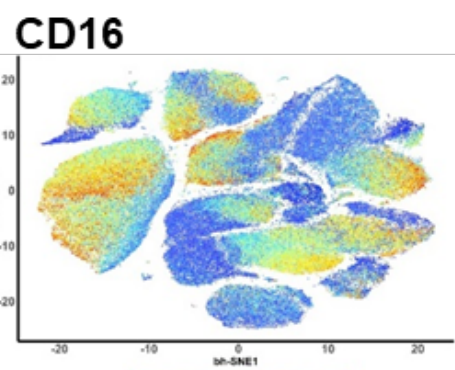

$\alpha 4 \beta 7$

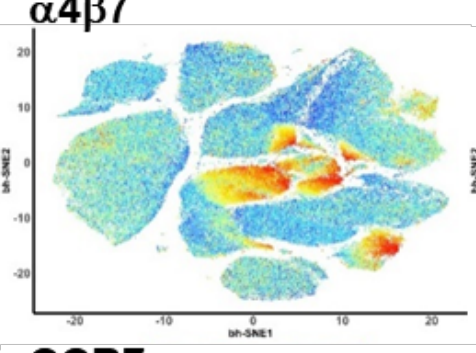

KIR2DL1/S1/S3/S5
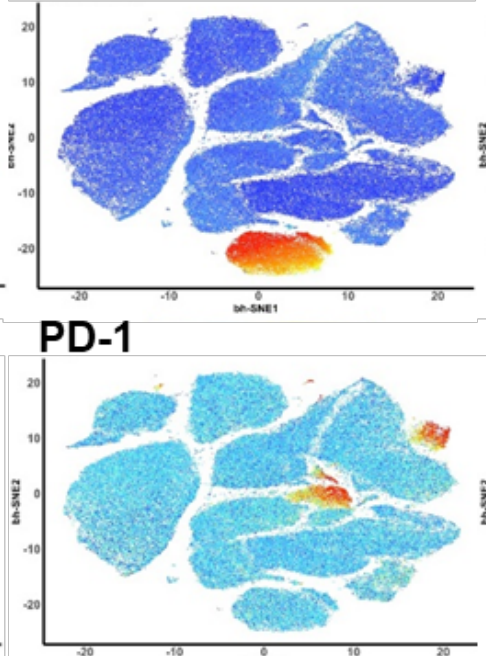

NKp46

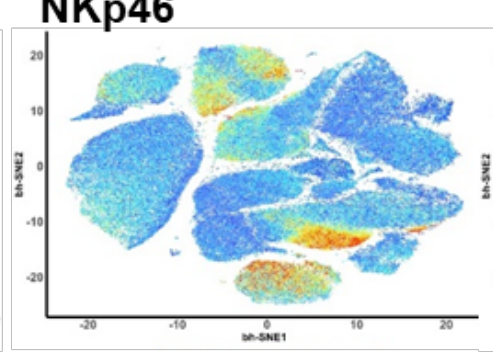

High

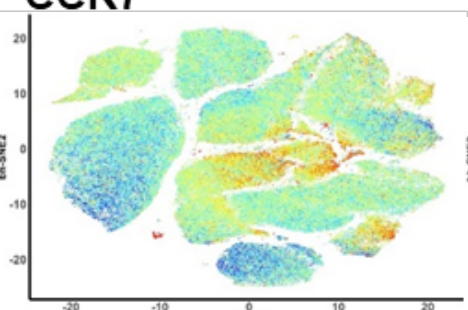

2B4

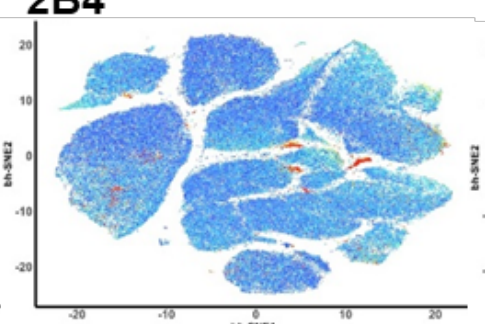

CD2

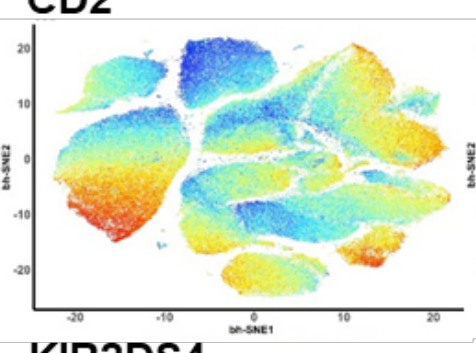

CCR5

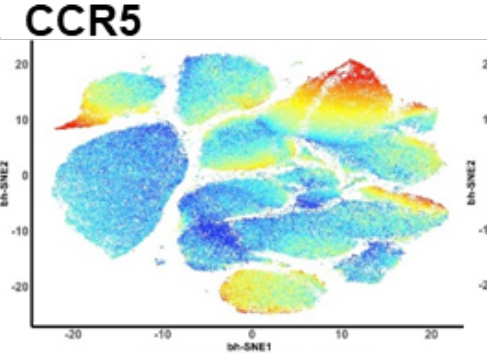

NKG2D

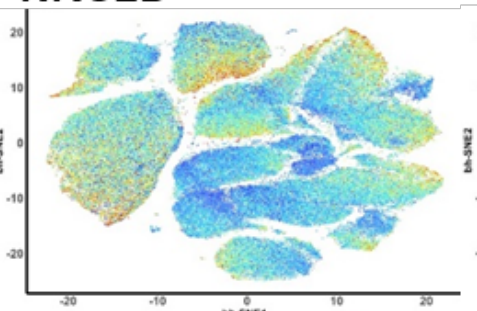

HLA-DR

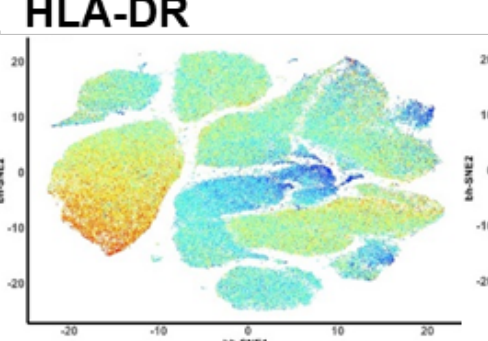

CD56

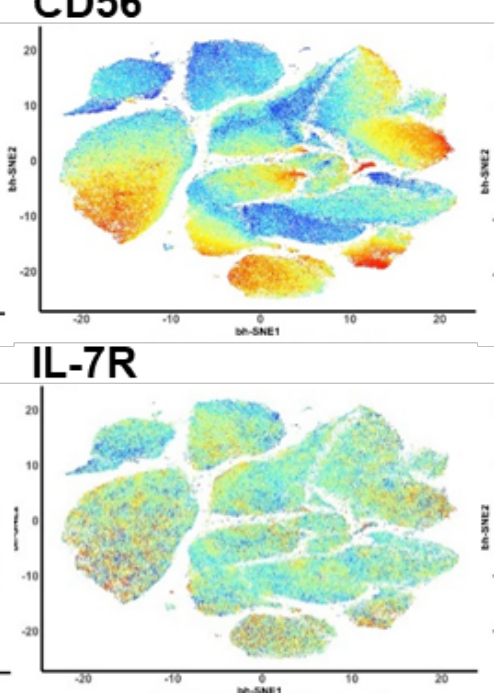

CD85j

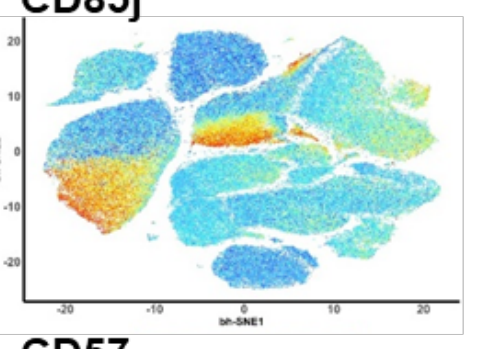

CD57

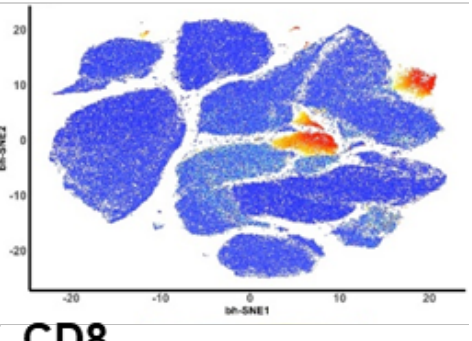

CD8

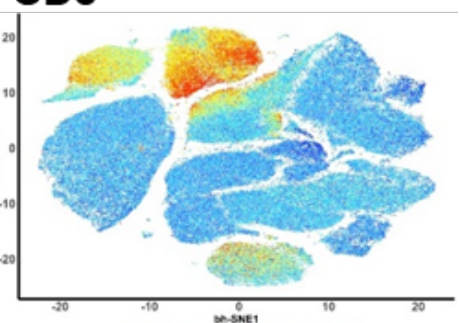

CXCR6

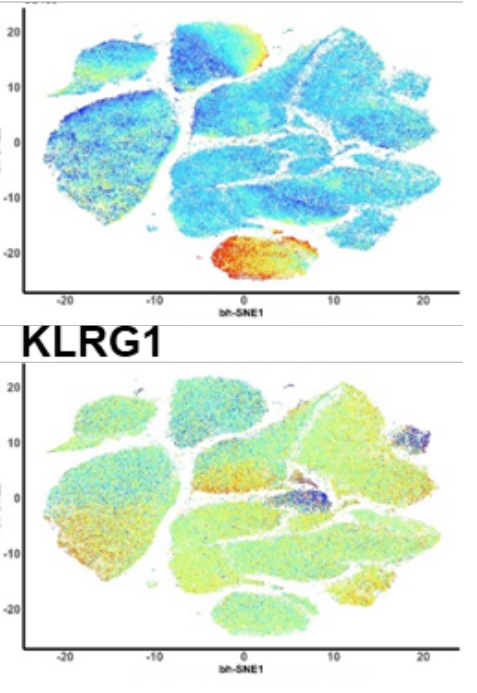


Supplementary Fig.6

a

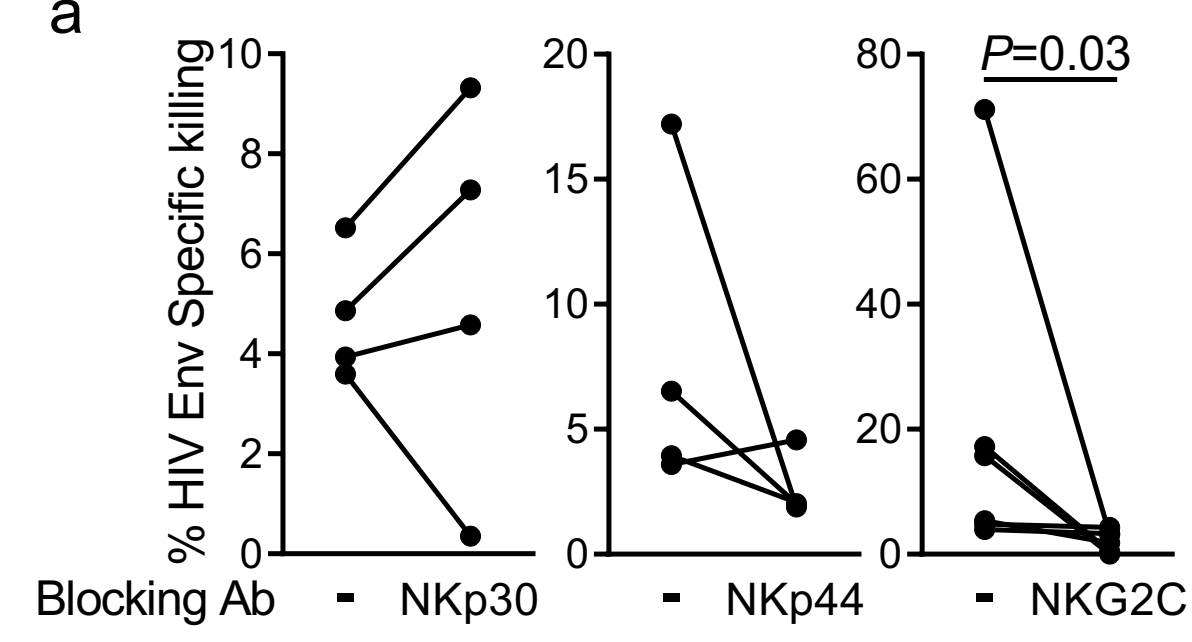

b

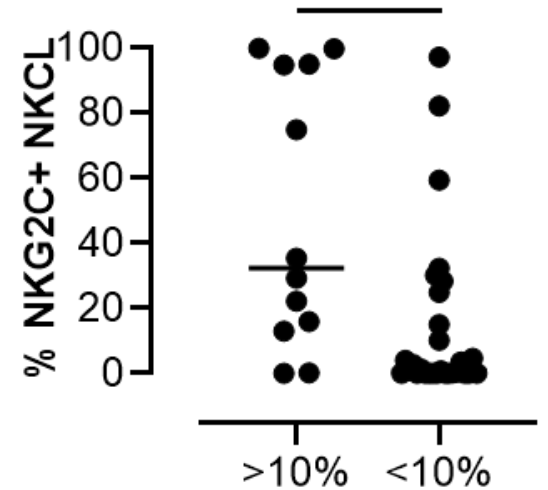

$\%$ Influenza-specific-killing 
